\title{
Droplet motion on inclined heterogeneous substrates
}

\author{
Nikos Savva ${ }^{1,2}$ and Serafim Kalliadasis ${ }^{2, \dagger}$ \\ ${ }^{1}$ Cardiff School of Mathematics, Cardiff University, Cardiff CF24 4AG, United Kingdom \\ ${ }^{2}$ Department of Chemical Engineering, Imperial College London, London SW7 2AZ, United Kingdom
}

(Received 7 December 2012; revised 5 April 2013; accepted 15 April 2013;

first published online 14 May 2013)

\begin{abstract}
We consider the static and dynamic behaviour of two-dimensional droplets on inclined heterogeneous substrates. We utilize an evolution equation for the droplet thickness based on the long-wave approximation of the Stokes equations in the presence of slip. Through a singular perturbation procedure, evolution equations for the location of the two moving fronts are obtained under the assumption of quasistatic dynamics. The deduced equations, which are verified by direct comparisons with numerical solutions to the governing equation, are scrutinized in a variety of dynamic and equilibrium settings. For example, we demonstrate the possibility for stick-slip dynamics, substrate-induced hysteresis, the uphill motion of the droplet for sufficiently strong chemical gradients and the existence of a critical inclination angle beyond which the droplet can no longer be supported at equilibrium. Where possible, analytical expressions are obtained for various quantities of interest, which are also verified by appropriate numerical experiments.
\end{abstract}

Key words: contact lines, drops, thin films

\section{Introduction}

Droplet motion is frequently encountered in our everyday lives but is also relevant to a broad range of applications. In some settings droplets must be avoided, e.g. car windscreens, solar panels, greenhouse covers and heat exchangers need to remain as dry as possible, whereas droplet retention is critical for the efficiency of several applications, such as the deposition of pesticides on plant foliage or in condensation processes. Not surprisingly a large number of studies have been devoted to droplets in various settings and configurations. Yet, several issues associated with droplet motion remain unresolved, particularly in relation to contact line behaviour on inclined heterogeneous substrates. In the present study, we analyse various static and dynamic aspects of hydrophilic, two-dimensional (2D) droplet dynamics on such substrates through a rigorous and systematic asymptotic analysis of the long-wave equations in the Stokes regime.

A common observation of a droplet on an inclined substrate is that it appears to remain stationary as the inclination angle is increased, until a critical inclination angle 
is reached beyond which the substrate can no longer support the droplet at equilibrium and it slides downhill at a nearly constant speed (rolling motion is also possible for sufficiently hydrophobic substrates; see Mahadevan \& Pomeau 1999; Richard \& Quéré 1999). By increasing the inclination angle even further, cusp formation is eventually observed at the rear of the droplet, which may lead to breakup due to instabilities and the shedding of droplets (Podgorski, Flesselles \& Limat 2001; Ben Amar, Cummings \& Pomeau 2003; Limat \& Stone 2004; Le Grand, Daerr \& Limat 2005; Snoeijer et al. 2005, 2007).

The ability of inclined substrates to retain the fluid without sliding is commonly attributed to contact angle hysteresis, i.e. the existence of a range of contact angles where the contact line appears to be immobile, thus observing different advancing and receding contact angles (Dussan 1979). As shown rigorously by Finn \& Shinbrot (1988), the droplet cannot be held at equilibrium on an inclined substrate in the absence of hysteresis, which is a consequence of the fact that the problem is overdetermined: the Young-Laplace equation that governs the free surface of the droplet is required to satisfy too many conditions. The problem becomes well-posed if the contact angle is allowed to vary along the contact line and some additional constraint is imposed, which is nevertheless chosen in an ad hoc manner. In theoretical investigations focusing on droplet equilibria, the authors typically either prescribe the shape of the contact line, which is assumed to be maintained up to and including the critical inclination angle (see, for example, Brown, Orr \& Scriven 1980; Lawal \& Brown 1982; Dussan \& Chow 1983; Popova 1983; Dussan 1985), or prescribe the variation of the contact angle along the contact line (see, for example, Larkin 1967; Rotenberg, Boruvka \& Neumann 1984). Alternative approaches have also been proposed to obtain equilibrium droplet shapes. These include a phenomenological energy barrier, which is assumed to be proportional to the contact area (Iliev 1997), the assumption that a thin liquid film is formed behind a drop on a hydrophilic substrate at the expense of gravitational energy (Roura \& Fort 2001) or the minimization of hysteresis for fixed droplet size and advancing contact angle, together with some phenomenological condition to ensure that convergence to non-physical solutions is avoided (Dimitrakopoulos \& Higdon 1999). It is apparent, however, that all of the above modelling approaches are applicable for rather specific conditions, thus limiting their usage in a more general setting and their overall predictive capabilities.

Apart from obtaining droplet shapes, the identification of conditions under which a droplet will eventually slide downhill has been the focus of many studies in the field. These conditions are essentially equivalent to determining the critical contact angle, $\phi_{\text {crit }}$, which is defined as the inclination angle for which the droplet is at the point of incipient motion, or, in other words, the maximum angle at which the droplet can be supported at equilibrium by the substrate. Macdougall \& Ockrent (1942) were the first to deduce $\phi_{\text {crit }}$ for $2 \mathrm{D}$ droplets using force-balance arguments,

$$
\cos \theta_{r}-\cos \theta_{a}=\frac{\rho g}{\sigma} A \sin \phi_{c r i t},
$$

where $A$ is the droplet area, $g$ is the gravitational acceleration, $\rho$ is the liquid density, $\sigma$ is the surface tension and $\theta_{r}$ and $\theta_{a}$ correspond to the receding and advancing contact angles, respectively. This formula was also derived a few years later by Frenkel (1948) through a minimization of the total energy of the system. Since then, a number of authors (see, for example Furmidge 1962; Dussan 1985; Quéré 1998; Miwa et al. 2000; ElSherbini \& Jacobi 2004a) proposed generalizations of (1.1) to account 
for three-dimensional (3D) or other effects. These amendments, which nevertheless still retain the basic functional form of (1.1), required the consideration of more phenomenological arguments, since the inclusion of other effects is considerably more complex, unless a number of simplifying assumptions are taken into account, such as weak hysteresis, stipulations about the substrate morphology, the droplet and/or contact-line shapes, etc.

Equation (1.1) and its different variants have been confirmed experimentally by many authors (see, e.g., Macdougall \& Ockrent 1942; Bikerman 1950; Furmidge 1962; Briscoe \& Galvin 1991; Extrand \& Kumagai 1995; Quéré 1998; Roura \& Fort 2001). However, it is important to emphasize that even though $\theta_{a}$ and $\theta_{r}$ in (1.1) are typically taken to be the advancing and receding contact angles measured on horizontal substrates, a number of authors considered different ways of determining them. For example, Krasovitski \& Marmur (2005) argued by geometric arguments that a distinction needs to be made between advancing/receding and the critical maximum/minimum contact angles, since the latter angles are expected to be dependent on the droplet volume. The experiments by ElSherbini \& Jacobi $(2004 a, b)$ showed that indeed the maximum/minimum angles depend on the droplet size, albeit rather weakly. This dependence, however, is also affected by the surface/liquid combination used in an experiment, as reported in the experiments by Pierce, Carmona \& Amirfazli (2008), in which a particular surface/liquid combination yielded significantly different results for $\cos \theta_{r}-\cos \theta_{a}$, depending on what pairs of angles were used in the calculation.

Recently, a number of experimental studies highlighted some issues related with measurements in an experimental setting. Pierce et al. (2008) reported statistically significant differences in critical inclination angle measurements among different experimental methods and droplet deposition techniques. More specifically, they found that the critical inclination angle measured on an already inclined substrate tended to be less than that measured on a substrate that was initially horizontal and inclined gradually afterwards, attributing their observations to differences in the droplet shapes occurred during the deposition phase of the experiment. Berejnov \& Thorne (2007) investigated in detail the transient dynamics as the inclination angle is increased, in which the contact line undergoes very small displacements due to the droplet becoming locally unstable and subsequently moves to a nearby metastable state. Tadmor et al. (2008) observed that the retention force that keeps the droplet pinned at the substrate tended to increase in time, thus requiring larger inclination angles to induce motion. These observations were explained in terms of the unbalanced normal component of Young's equation which slowly deforms the substrate, which in turn introduces additional energy barriers (we note, however, that a recent effort by Pereira \& Kalliadasis 2012 on describing contact lines using density-functional theory shows that the normal component of Young's equation is in fact balanced by the disjoining pressure which is in turn related to the chemical potential of the system and its distance from its saturation value). Apart from the influence of contact line history on the measurement of the critical inclination angle, noteworthy are also the experiments by Yadav et al. (2008) which indicated that the retention force is also a weak function of the droplet size.

The studies on the dynamic droplet behaviour, both experimental and theoretical, placed greater emphasis on the regime where the droplet descends steadily down the incline and its shape does not change in time. In addition to the studies that investigated in detail the cusp that may develop at the rear of the droplet and its instabilities (mentioned above), the work by Kim, Lee \& Kang (2002) focused 
specifically on the steady, slow motion of rounded droplets at small inclination angles, deducing a phenomenological scaling law to explain their observations (see also Podgorski et al. 2001; Le Grand et al. 2005, in which both rounded and cusped droplets are investigated). To address the dynamic problem numerically, a broad range of methodologies were utilized, ranging from precursor film models (e.g. Thiele et al. 2001, 2002; Koh et al. 2009), Lattice Boltzmann simulations (e.g. Dupuis \& Yeomans 2006), to smoothed particle hydrodynamics (e.g. Das \& Das 2009) and molecular dynamics simulations (e.g. Servantie \& Müller 2008; Hong, Ha \& Balachandar 2009 for a horizontal body force, which is reminiscent of the gravitational component parallel to the inclined substrate, or equivalently for vertical substrates). Regarding the analysis of 2D droplets, we note the study of Hocking (1981), in which he considered the linearized dynamics with slip and imposed hysteresis effects, and the study by Durbin (1988), who considered a yield stress boundary condition to justify the pinning of the droplet. 3D droplets are considerably more difficult to examine analytically, unless some assumptions are made to simplify the analysis. Perhaps the only systematic analyses on 3D droplets are those presented by Dussan \& Chow (1983) and Dussan (1985), in which they assumed hysteresis with imposed speed-contact angle relations and that the contact line was parallel sided with circular arcs at the front and rear of the droplet.

Even though substrate heterogeneities are viewed by many authors as a plausible source for contact angle hysteresis (see Bonn et al. 2009, and the references therein), droplets on inclined heterogeneous substrates have received comparatively less attention, with the exception of a handful of studies. These works were primarily experimental in nature although a few contained some analysis based on rather simplistic energy arguments. Bikerman (1950) observed that the critical inclination angle increases with substrate roughness, arguing that the resistance to sliding is affected in a similar manner as it occurs in solids. Extrand \& Kumagai (1995), who examined the validity of (1.1) for surfaces of different roughness, argued that hysteresis cannot be attributed solely to substrate roughness, but also to chemical heterogeneities, since they observed that substrates of similar roughness characteristics exhibited markedly different levels of hysteresis. Miwa et al. (2000) reported experiments with hydrophobic needle-like structured substrates where they observed that the critical angle decreased with increasing contact angle. They also deduced an equation for the critical inclination angle based on energy considerations to explain qualitatively their observations, whose validity was later questioned by Roura \& Fort (2002). Sommers \& Jacobi (2006, 2008) performed a series of experiments with microgrooved substrates, where they observed that the motion was greatly influenced by the characteristics of the heterogeneities and their orientation relative to gravity. More specifically, they observed reduced critical angles when the groove-spacing is sufficiently small. This was attributed to the formation of small air pockets, which act as cushions that help the droplet in its downhill motion. When the grooves are sufficiently separated so that according to their arguments no air pockets form, they found that the critical inclination angle was largest when the groove orientation was normal to the component of gravity acting parallel to the substrate and smallest when it was parallel, as also observed experimentally by Yoshimitsu et al. (2002) and confirmed numerically by Hyväluoma et al. (2007). Experiments with line-patterned, chemically heterogeneous substrates revealed a similar behaviour (see Morita et al. 2005; Suzuki et al. 2008). The results of these studies is a clear manifestation that substrate heterogeneities may act as energy barriers resisting droplet motion. Such energy barriers is a prerequisite for equilibrium even when nanoscopic 
effects are taken into account, as demonstrated by Berim \& Ruckenstein (2008) using density-functional theory. However, heterogeneities can also assist the uphill motion, as shown by Chaudhury \& Whitesides (1992), where they reported that sufficiently strong wettability gradients are able to move the droplets uphill, against gravity.

The aim of the present study is to investigate the statics and dynamics of a $2 \mathrm{D}$ droplet on a heterogeneous inclined substrate, by examining carefully the combined effects of gravity, substrate topography and chemical heterogeneities, in an attempt to rationalize qualitatively some of the experimental observations. We do acknowledge some of the limitations of our model, namely the fact that we focus on small contact angles so that the long-wave approximation may be employed together with the twodimensionality of the droplet which does not capture some 3D features (e.g. the formation of cusps at the rear). But these are not deterrents for our work, which is the first to addresses the problem through a rigorous analytical-numerical investigation based on first principles and where neither hysteresis nor an empirical spreading law are imposed a priori. The analysis focuses in the quasi-static dynamics and is based on a systematic matching procedure where the solutions in the liquid bulk are asymptotically matched to those near the contact lines. This part of our work offers effectively a generalization of the previous work on droplet motion on horizontal substrates including heterogeneous ones (see, e.g., Hocking 1983; Savva \& Kalliadasis 2009, 2012; Vellingiri, Savva \& Kalliadasis 2011).

Specifically, in $\$ 2$ we present in detail the model assumptions, governing equation and the appropriate boundary conditions and in $\S 3$ we obtain via asymptotic matching evolution equations for the location of the two droplet fronts in the form of a pair of coupled integrodifferential equations (IDEs). In the sections that follow we examine the obtained equations in a variety of settings. In $\S 4$ we briefly discuss the case of having an ideal, homogenous substrate, obtaining analytically an expression for the speed of descent in the limit of weakly inclined substrates. This is followed by $\S 5$ where we investigate the influence of substrate topography and chemical heterogeneities with the help of analysis, computations and phase-plane arguments. In $\S 6$ we study the critical angle and how it is affected by substrate heterogeneities. We close with concluding remarks and discussion in $§ 7$.

\section{Model}

Consider a 2D droplet (hereinafter referred to simply as a 'droplet') on a heterogeneous substrate, which is inclined at an angle $\phi$ to the horizontal. The droplet lies on the $X-Z$ plane and has thickness $H(X, T)$ at time $T$. The substrate, whose profile is prescribed by $S(X)$, which describes topographical variation, is also chemically heterogeneous, so that there exist local variations in the local equilibrium contact angle, given by $F(X)$. In our model we neglect inertial effects and consider the limit of small contact angles, i.e. $F(X) \ll 1$, so that we may employ the long-wave approximation in the Stokes regime. We further assume the presence of slip along the substrate, so that the velocity in the $X$ direction, $U(X, Z)$, satisfies the condition

$$
\left.U\right|_{Z=S(X)}=\left.\Lambda \partial_{Z} U\right|_{Z=S(X)},
$$

where $\Lambda$ corresponds to the slip length. Even though it is natural to expect $\Lambda$ to vary with the chemical heterogeneities on the substrate, we have chosen to keep it constant in order to avoid having to introduce additional degrees of freedom into our problem, noting also that this assumption was also made in related studies (see, for example, Greenspan 1978; Vellingiri et al. 2011). In addition, as it will turn out from 
our analysis in $\S 3$, the dependence of the dynamics on $\Lambda$ is logarithmic, i.e. weak in general, especially if the order of magnitude of $\Lambda$ is not altered by heterogeneities.

By putting everything together, we obtain an evolution equation for $H(X, T)$, based on the height-averaged continuity equation,

$$
\partial_{T} H+\partial_{X}(H \bar{U})=0
$$

where $\bar{U}$ is the height-averaged velocity along the $X$-axis. In the long-wave approximation, $\bar{U}$ becomes

$$
\bar{U}=\frac{H(H+3 \Lambda)}{3 \mu}\left[\sigma \partial_{X}^{3}(H+S)-\rho g \cos \phi \partial_{X}(H+S)+\rho g \sin \phi\right]
$$

where $g$ is the gravitational acceleration, with $\rho, \sigma$ and $\mu$ being the density, surface tension and viscosity of the fluid, respectively. The coupling of contact line dynamics with long-wave models, such as (2.2) with (2.3), has a long history dating back to the studies of Greenspan (1978) and Hocking (1981), but it is also important to note that the same set of equations governs the motion of thin films flowing down an inclined plane (see Buckingham, Shearer \& Bertozzi 2003; Kondic 2003, for more details on the derivation), which is a subject that has also been widely investigated by many authors since the seminal work of Huppert (1982). In this context, the droplet we consider can be viewed as a liquid ridge/rivulet which is known to be prone to instabilities in the transverse direction (see, e.g., Silvi \& Dussan 1985; Troian et al. 1989; Bertozzi \& Brenner 1997; López, Miksis \& Bankoff 1997; Hocking 1990; Kalliadasis 2000; Diez \& Kondic 2001; Kondic 2003; Diez, González \& Kondic 2012). Here we neglect any motions that may give rise to instabilities and focus on the dynamics of a contact line which is uniform in the transverse direction. This simplifying assumption is a rather common one and has been invoked in numerous related studies in the past (see, e.g., Hocking 1981; Thiele et al. 2001, 2002; Savva \& Kalliadasis 2009, 2012; Vellingiri et al. 2011). It is also important to emphasize that film-like shapes can also be observed in solutions to (2.2) with (2.3) in the limit when gravity dominates capillarity, which causes the flattening of the droplet in the middle and the development of a pronounced bulge at the advancing edge (see also $\$ 4$ ). This gravity-dominated regime, however, is beyond the scope of the present study, and it requires a different treatment from that considered here.

The problem is made non-dimensional by introducing the lowercase variables

$$
x=\frac{X}{L}, \quad h=\frac{H}{L \tan \alpha_{s}}, \quad s=\frac{S}{L \tan \alpha_{s}}, \quad t=\frac{T}{\tau}, \quad \lambda=\frac{3 \Lambda}{L \tan \alpha_{s}}, \quad f=\frac{F}{\tan \alpha_{s}}
$$

where $\alpha_{s}$ is some reference contact angle, $\tau=3 \mu L /\left(\sigma \tan ^{3} \alpha_{s}\right)$ is the characteristic time scale of the problem and $L$ is its corresponding length scale, defined in terms of the cross-sectional area of the droplet, $A$, as $L=\sqrt{A \cot \alpha_{s}}$. This change of variables transforms (2.2) and (2.3) to

$$
\partial_{t} h+\partial_{x}\left\{h^{2}(h+\lambda)\left[\partial_{x}^{3}(h+s)-w_{1} \partial_{x}(h+s)+w_{2}\right]\right\}=0,
$$

where $w_{1}$ and $w_{2}$ are $O(1)$ parameters dependent on the Bond number, $B o=\rho g L^{2} / \sigma$, and are defined as

$$
w_{1}=B o \cos \phi \quad \text { and } \quad w_{2}=B o \sin \phi \cot \alpha_{s} .
$$




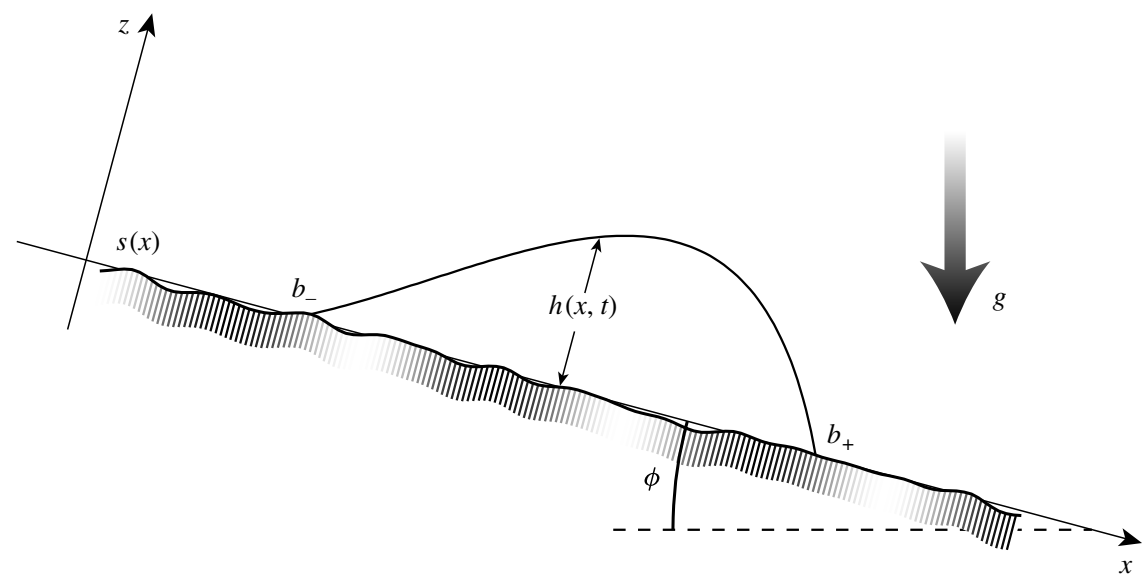

FIGURE 1. 2D droplet on a heterogeneous substrate inclined at an angle $\phi$ to the horizontal. In non-dimensional units, the substrate profile is given by $s(x)$ and the droplet thickness is given by $h(x, t)$, with its contact points located at $x=b_{ \pm}(t)$. The local contact angle variations along the substrate are prescribed by $f(x)$. The hatches below the substrate profile are used to show the variations of the contact angle. They are coloured in shades of grey between the maximum (white) and minimum (black) values of $f(x)$ corresponding to the portion of the substrate shown. The acceleration due to gravity, $g$, is vertically downward.

In what follows, we consider solutions to (2.5) subject to the constant-area constraint,

$$
\int_{b_{-}}^{b_{+}} h(x, t) \mathrm{d} x=1
$$

and conditions imposed at the right and left contact points, $x=b_{ \pm}(t)$ (see figure 1), i.e.

$$
\begin{gathered}
h=0, \\
\partial_{x} h=\mp \alpha_{ \pm},
\end{gathered}
$$

where the quantities $\alpha_{ \pm}$originate from the geometric constraints that fix the angles the free surface makes with the substrate to those prescribed by the local equilibrium contact angles, $f\left(b_{ \pm}\right)$, and are given by

$$
\alpha_{ \pm}=f\left(b_{ \pm}\right) \frac{1+\tan ^{2} \alpha_{s} s_{ \pm}^{\prime 2}}{1 \pm \tan ^{2} \alpha_{s} s_{ \pm}^{\prime}},
$$

where we set $s_{ \pm}^{\prime}=\left.\partial_{x} s\right|_{x=b_{ \pm}}$(see Savva \& Kalliadasis 2009). Equation (2.8) contains $O\left(\tan ^{2} \alpha_{s}\right)$ terms that are small since $\tan \alpha_{s} \ll 1$ by assumption, but are nevertheless retained to exactly enforce the constant-contact-angle conditions. It is apparent, however, that their effect is generally small. The profiles for the chemical and topographical heterogeneities, i.e. $f(x)$ and $s(x)$, respectively, can be arbitrary provided that they are continuous and their variations occur at length scales that are longer than the slip length, $\lambda \ll 1$. However, to be consistent with the assumption of the long-wave approximation and (2.5), we must further require that: (i) $\partial_{x}^{4} s$ is continuous; (ii) $f(x)>0$; and (iii) both $f(x)$ and $\partial_{x} s$ are at most $O(1)$ (Savva \& Kalliadasis 2009; Vellingiri et al. 2011). 
Even though solutions to (2.5) subject to (2.7) may be obtained accurately by an appropriate numerical scheme (see e.g. Savva \& Kalliadasis 2009), the analysis that follows facilitates greatly the extraction of various qualitative features related to the problem. Following a rigorous and systematic asymptotic procedure, which was originally introduced by Hocking (1983) for axisymmetric droplet spreading over horizontal ideal substrates, the ultimate aim is to obtain a set of evolution equations for the two moving fronts, $b_{ \pm}(t)$. Such equations may be analysed further to look for features that are not easily accessible by a brute force numerical approach, particularly the visualization of solutions to (2.5) on a phase plane, an impossible task for the infinite-dimensional dynamical system corresponding to (2.5).

\section{Analysis}

When $\lambda \ll 1$, we have a clear separation of length scales, with a bulk/outer region where the effects of slip are negligible, and two inner regions in the vicinity of the contact lines, in which slip is important. Within these inner regions, the slope of the free surface changes abruptly from a local equilibrium contact angle to the apparent contact angle in the bulk. Hence, based on earlier analyses (see, e.g., Hocking 1983; Savva \& Kalliadasis 2009, 2012; Vellingiri et al. 2011), we treat the outer region in the bulk with the two inner regions near the contact lines separately to obtain the asymptotic forms of $\partial_{x} h$ and, through a set of matching conditions, we can deduce equations for the time derivatives of the two moving fronts, $\dot{b}_{ \pm}=\mathrm{d} b_{ \pm} / \mathrm{d} t$. For this matching procedure to be successful, however, we must require slow spreading dynamics so that $\left|\dot{b}_{ \pm}\right| \ll 1$ and the assumption of quasi-static motion holds.

\subsection{Outer region}

Equation (2.5) subject to the conditions (2.7) is essentially a free boundary problem where the location of the contact points is determined as part of the solution. To analyse the outer-region dynamics, we transform the problem to one with fixed boundaries, by introducing the variable change

$$
x=\frac{b_{+}-b_{-}}{2} y+\frac{b_{+}+b_{-}}{2}
$$

that maps the physical domain $b_{-}(t) \leqslant x \leqslant b_{+}(t)$ to the interval $-1 \leqslant y \leqslant+1$. In the outer region, slip effects may be neglected so that (2.5) together with (3.1) simplifies to

$$
\partial_{t} h-\frac{\dot{b}_{+}(1+y)+\dot{b}_{-}(1-y)}{2 d} \partial_{y} h+\frac{1}{d^{4}} \partial_{y}\left\{h^{3}\left[\partial_{y}^{3}(h+s)-d^{2} w_{1} \partial_{y}(h+s)+d^{3} w_{2}\right]\right\}=0,
$$

where we used $d=\left(b_{+}-b_{-}\right) / 2$ to denote the droplet 'radius'. From our discussion above, the outer region cannot capture the dynamics near the contact line and, hence, (3.2) is solved subject to $(2.7 a, b)$. Assuming that $\left|\dot{b}_{ \pm}(t)\right| \ll 1$, we invoke a quasi-static expansion of the form

$$
h=h_{0}\left(y ; b_{ \pm}(t)\right)+h_{1}\left(y ; b_{ \pm}(t), \dot{b}_{ \pm}(t)\right)+\cdots
$$

where we take $h_{0} \gg h_{1}$ and $h_{1}$ is linear in $\dot{b}_{ \pm}(t)$. Plugging (3.3) in (3.2) and collecting $O$ (1) terms yields an equation for $h_{0}$

$$
\partial_{y}^{3}\left(h_{0}+s\right)-d^{2} w_{1} \partial_{y}\left(h_{0}+s\right)+d^{3} w_{2}=0,
$$


which must satisfy the conditions $h_{0}( \pm 1)=0$ and $\int_{-1}^{+1} h_{0}(y) \mathrm{d} y=2 / d$. Equation (3.4) is a linear ordinary differential equation, whose solution is given analytically as

$$
\begin{aligned}
h_{0}(y)= & \frac{(1 / 2 d)+\bar{s}-\left(s_{+}+s_{-}\right) / 2}{\cosh k-k^{-1} \sinh k}(\cosh k-\cosh k y)+\frac{w_{2} d^{3}}{k^{2}}\left(y-\frac{\sinh k y}{\sinh k}\right) \\
& +\frac{1}{2}\left(s_{+}+s_{-}\right)+\frac{1}{2}\left(s_{+}-s_{-}\right) \frac{\sinh k y}{\sinh k}-s(\mathrm{~d} y+\ell),
\end{aligned}
$$

where $\ell=\left(b_{+}+b_{-}\right) / 2, k=d \sqrt{w_{1}}, \bar{s}=\frac{1}{2} d^{-1} \int_{b_{-}}^{b_{+}} s(x) \mathrm{d} x$ and $s_{ \pm}=s\left(b_{ \pm}\right)$. For the nextorder term, take $\partial_{t} h=\dot{b}_{+} \partial_{b_{+}} h_{0}+\dot{b}_{-} \partial_{b_{-}} h_{0}+\cdots$ so that the equation for $h_{1}$ becomes

$$
\partial_{y}^{3} h_{1}-d^{2} w_{1} \partial_{y} h_{1}=-\frac{d^{4} D(y)}{h_{0}^{3}}
$$

where

$$
\begin{aligned}
D(y) & =\int\left[\dot{b}_{+}\left(\partial_{b_{+}} h_{0}-\frac{1+y}{2 d} \partial_{y} h_{0}\right)+\dot{b}_{-}\left(\partial_{b_{-}} h_{0}-\frac{1-y}{2 d} \partial_{y} h_{0}\right)\right] \mathrm{d} y \\
& =q_{+}(y) \theta_{+} \dot{b}_{+}+q_{-}(y) \theta_{-} \dot{b}_{-},
\end{aligned}
$$

with

$$
\begin{aligned}
q_{ \pm}(y) & =\frac{1}{2}\left(\frac{\cosh k y-\cosh k}{k \sinh k} \pm \frac{\sinh k y-y \sinh k}{k \cosh k-\sinh k}\right) \\
\theta_{ \pm} & =\frac{k^{2}}{d} \frac{(1 / 2 d)+\bar{s}-\left(s_{+}+s_{-}\right) / 2}{k \operatorname{coth} k-1} \pm \frac{w_{2} d^{2}}{k^{2}}(k \operatorname{coth} k-1) \mp k \frac{s_{+}-s_{-}}{2 d} \operatorname{coth} k \pm s_{ \pm}^{\prime} .
\end{aligned}
$$

It should be noted that $\theta_{ \pm}$can be interpreted as the mesoscopic contact angles, found from the slopes of the leading-order outer solution, $\theta_{ \pm}=\left.\mp \partial_{x} h_{0}\right|_{x=b_{ \pm}}=\left.\mp d^{-1} \partial_{y} h_{0}\right|_{y= \pm 1}$. When we have small-scale topography, the apparent contact angle, $\theta_{a p p \pm}$ is obtained by evaluating (3.9) when $s(x)=0$, namely

$$
\theta_{a p p \pm}=\frac{k^{2}}{2 d^{2}(k \operatorname{coth} k-1)} \pm \frac{w_{2} d^{2}}{k^{2}}(k \operatorname{coth} k-1) .
$$

Note also that (3.5) is a physically acceptable leading-order outer solution only within a range of parameters. It is valid in the regime when capillarity is dominant, i.e. for comparatively small $B o$. For larger $B o$, gravity flattens the droplet considerably as noted earlier, whereas (3.5) predicts non-physical intersections of the droplet free surface with the substrate, thus making $\theta_{-}$negative. The asymptotics of this regime must be treated differently, following some of the ideas outlined by Hocking (1983). To get a rough estimate of the regime of applicability of our asymptotic theory, we look at the homogeneous (chemically and topographically) limit and $\theta_{a p p-}$ which must always be positive, i.e. when

$$
w_{2} \ll \frac{k^{4}}{2 d^{4}(k \operatorname{coth} k-1)^{2}} .
$$

When $\phi=0$, i.e. for horizontal substrates, the right-hand side of (3.11) attains the value 2 for a droplet at equilibrium. Hence, a reasonably accurate order-of-magnitude 
estimate for the limits of applicability of our theory is the requirement:

$$
\frac{B o \sin \phi}{\tan \alpha_{s}} \ll 2 \text {. }
$$

However, (3.12) should not be viewed as a strict bound, since it may be significantly affected by substrate heterogeneities.

The higher-order correction, $h_{1}(y)$, cannot be obtained analytically from (3.6), but in order to complete the matching procedure it suffices to determine its asymptotic behaviour as the contact lines are approached, namely as $y \rightarrow \pm 1$. It is easy to verify from (3.6) that the third derivative of $h_{1}$ is singular as $y \rightarrow \pm 1$ and behaves like

$$
\partial_{y}^{3} h_{1} \sim \frac{d \dot{b}_{ \pm}}{\theta_{ \pm}^{2}(1 \mp y)^{2}}+O\left((1 \mp y)^{-1}\right)
$$

Integrating twice with respect to $y$ yields the leading-order behaviour of $\partial_{y} h_{1}$ as $y \rightarrow \pm 1:$

$$
\partial_{y} h_{1} \sim-\dot{b}_{ \pm} \frac{d}{\theta_{ \pm}^{2}}\left[\ln \frac{e}{2}(1 \mp y)+\beta_{ \pm}\right],
$$

where $\beta_{ \pm}$are (time-dependent) parameters to be found subject to homogeneous conditions for $h_{1}$, namely

$$
h_{1}( \pm 1)=0 \quad \text { and } \quad \int_{-1}^{+1} h_{1}(y) \mathrm{d} y=0 .
$$

The procedure to determine $\beta_{ \pm}$involves the multiplication of both sides of (3.6) by $q_{ \pm}(y)$ and integration with respect to $y$ from -1 to +1 , to which we apply successive integrations by parts, conditions (3.15) and the asymptotic form of $h_{1}$, (3.14). After some algebra, we obtain

$$
\beta_{ \pm}=\int_{-1}^{+1}\left[\frac{1}{1 \mp y}-\frac{d^{3} \theta_{ \pm}^{2} q_{ \pm}(y)}{h_{0}^{3} \dot{b}_{ \pm}} D(y)\right] \mathrm{d} y .
$$

Having determined $\beta_{ \pm}$, we can express the leading-order asymptotic behaviour of $\partial_{x} h$ as the contact points are approached in terms of the physical coordinates,

$$
\mp \partial_{x} h \sim \theta_{ \pm} \pm \frac{\dot{b}_{ \pm}}{\theta_{ \pm}^{2}}\left[\ln \left(\mp e \frac{x-b_{ \pm}}{2 d}\right)+\beta_{ \pm}\right] \quad \text { as } x \rightarrow b_{ \pm} .
$$

The unknowns $\dot{b}_{ \pm}$are to be determined by a condition obtained from matching (3.17) with the corresponding solutions of the inner regions. This is carried out within an appropriate overlap region, in which the $x$-dependent logarithmic terms are of higherorder compared with the $O\left(\dot{b}_{ \pm}^{0}\right)$ term, $\theta_{ \pm}$.

\subsection{Inner regions}

As noted previously, the details of the solution near the contact points cannot be resolved solely by considering the outer region due to the disparity of length scales present in the problem, namely the microscopic slip length and the droplet radius. Near the contact points we need to account for slip effects, since these eventually provide the necessary mechanism for contact line motion. Hence, to probe into these regions, in which $h= \pm\left(x-b_{ \pm}\right)=O(\lambda)$, we introduce the stretched variables

$$
\xi_{ \pm}=\mp\left(x-b_{ \pm}\right) \alpha_{ \pm} / \lambda \text { and } \Phi_{ \pm}=h / \lambda \text {, }
$$


which transform (2.5) into

$$
\pm \dot{b}_{ \pm} \partial_{\xi \pm} \Phi_{ \pm}+\alpha_{ \pm}^{3} \partial_{\xi_{ \pm}}\left[\Phi_{ \pm}^{2}\left(\Phi_{ \pm}+1\right) \partial_{\xi_{ \pm}}^{3} \Phi_{ \pm}\right]=0
$$

where we retained only the $O\left(\lambda^{0}\right)$ terms and neglected the contributions due to gravity and topography, by taking $\lambda^{2} B o \ll 1$ and $\lambda\left|\partial_{x}^{3} s\right| \ll 1$, respectively. The latter is consistent with our original assumption that substrate variations are assumed to occur at length scales that are longer than the slip length, $\lambda$. The substrate topography, however, can still influence, albeit weakly, the inner-region dynamics through the boundary condition (2.7c). Equation (3.19) has been considered previously by a number of authors (see, e.g., Hocking 1983; Savva \& Kalliadasis 2009, 2012; Vellingiri et al. 2011), but we mention here the main results for completeness. By considering, like before, quasi-steady dynamics, we have an expansion of the form

$$
\Phi_{ \pm}=\xi_{ \pm}+\tilde{\Phi}_{ \pm}\left(\xi_{ \pm} ; \dot{b}_{ \pm}, \alpha_{ \pm}\right)+\cdots
$$

in which we have assumed a wedge-shaped free surface to leading order and $\tilde{\Phi}_{ \pm} \ll \xi$. The equation for $\tilde{\Phi}_{ \pm}$becomes

$$
\partial_{\xi_{ \pm}}^{3} \tilde{\Phi}_{ \pm}=\mp \frac{\dot{b}_{ \pm} / \alpha_{ \pm}^{3}}{\xi_{ \pm}\left(\xi_{ \pm}+1\right)},
$$

which is solved subject to the conditions $\tilde{\Phi}_{ \pm}=\partial_{\xi_{ \pm}} \tilde{\Phi}_{\xi_{ \pm}}=0$ at $\xi_{ \pm}=0$ and $\tilde{\Phi}_{ \pm} / \xi_{ \pm}^{2} \rightarrow 0$ as $\xi_{ \pm} \rightarrow \infty$. The slope of the solution is

$$
\partial_{\xi_{ \pm}} \tilde{\Phi}_{ \pm}=\mp \frac{\dot{b}_{ \pm}}{\alpha_{ \pm}^{3}}\left[\left(\xi_{ \pm}+1\right) \ln \left(\xi_{ \pm}+1\right)-\xi_{ \pm} \ln \xi_{ \pm}\right]
$$

whose asymptotic behaviour is

$$
\partial_{\xi_{ \pm}} \tilde{\Phi}_{ \pm} \sim \mp \frac{\dot{b}_{ \pm}}{\alpha_{ \pm}^{3}}\left(\ln \xi_{ \pm}+1\right) \quad \text { as } \xi_{ \pm} \rightarrow+\infty .
$$

From the above, we obtain the asymptotic slope of the free surface,

$$
\mp \partial_{x} h \sim \alpha_{ \pm} \pm \frac{\dot{b}_{ \pm}}{\alpha_{ \pm}^{2}}\left[\ln \left(\mp \alpha_{ \pm} \frac{x-b_{ \pm}}{\lambda}\right)+1\right] \quad \text { as } \mp \frac{x-b_{ \pm}}{\lambda} \rightarrow \infty,
$$

as expressed in terms of the outer variables, which is to be matched with the outer solution as the contact points are approached.

\subsection{Matching}

As first pointed out by Hocking (1983), for the problem of a droplet on a homogeneous horizontal substrate, the outer and inner regions do not generally match within the appropriate overlap regions, thus necessitating the presence of intermediate regions, located between the inner and outer ones. The sole purpose of these additional considerations, however, is to rigorously justify why matching can be carried out for $\left(\partial_{x} h\right)^{3}$ instead of $\partial_{x} h$ (Savva \& Kalliadasis 2009). The same observations can be made for the outer (3.17) and inner solutions (3.24) obtained above. Hence, if we consider the cubes of (3.17) and (3.24), we can now match their asymptotic forms, which upon elimination of the $x$-dependent logarithmic terms yields

$$
\pm \dot{b}_{ \pm}\left[\ln \frac{2 d \alpha_{ \pm}}{\lambda}-\beta_{ \pm}\right]=\theta_{ \pm}^{3}-\alpha_{ \pm}^{3} .
$$


From (3.25) we see that $\dot{b}_{ \pm}$must be $O(1 /|\ln \lambda|)$ for matching to be most effective. However, it has been observed in previous studies (Savva \& Kalliadasis 2009, 2012; Vellingiri et al. 2011) that the applicability of equations, such as (3.25), resulting from the matching conditions typically extends both to higher and lower spreading rates. Equations (3.25) may be readily solved for $\dot{b}_{ \pm}$:

$$
\dot{b}_{ \pm}= \pm \frac{\delta_{ \pm} G_{\mp}+\delta_{\mp} \theta_{ \pm} G_{0}}{G_{-} G_{+}-\theta_{-} \theta_{+} G_{0}^{2}}
$$

with

$$
\begin{aligned}
G_{ \pm} & =\ln \frac{2 d \alpha_{ \pm}}{\lambda}-\int_{-1}^{+1}\left[\frac{1}{1 \mp y}-\frac{d^{3} \theta_{ \pm}^{3} q_{ \pm}^{2}}{h_{0}^{3}}\right] \mathrm{d} y, \\
G_{0} & =\theta_{+} \theta_{-} d^{3} \int_{-1}^{+1} \frac{q_{+} q_{-}}{h_{0}^{3}} \mathrm{~d} y, \\
\delta_{ \pm} & =\frac{1}{3}\left(\theta_{ \pm}^{3}-\alpha_{ \pm}^{3}\right) .
\end{aligned}
$$

This system of IDEs is of similar structure to those obtained for horizontal heterogeneous substrates (Savva \& Kalliadasis 2009, 2012; Vellingiri et al. 2011), which are obviously special cases to the more general configuration considered here. Even though the dynamical system (3.26) possesses a somewhat complex structure, one has to bear in mind that with the above analysis we achieved the reduction of a nonlinear, free boundary problem to a much more tractable set of equations that, as we shall demonstrate, can reliably reproduce the behaviour of the original problem, namely (2.5) with conditions (2.7). In addition, the utilization of this simpler system of equations, particularly in a phase-plane analysis, has been proven in previous studies (Savva \& Kalliadasis 2009, 2012; Vellingiri et al. 2011) to be a rather useful tool in scrutinizing many qualitative aspects of the full problem. As a result, the following sections are devoted in solving both the full (2.5) and reduced problems (3.26) in a more detailed investigation of the combined effects of substrate heterogeneities and inclination angle.

\section{Ideal substrates}

From (3.26), it is clear that the droplet reaches equilibrium, i.e. $\dot{b}_{ \pm}=0$, when $\delta_{ \pm}=0$. This in turn implies that the droplet can attain equilibrium when the mesoscopic contact angles, $\theta_{ \pm}$, become equal to the local microscopic contact angles, $\alpha_{ \pm}$. While such a situation can possibly arise for heterogeneous substrates, it is impossible for the droplet to be at equilibrium when it spreads on an inclined ideal substrate that is homogeneous (chemically and topographically), namely when $s(x)=0$ and $f(x)=\alpha_{ \pm}=1$. This is because when we have $w_{2} \neq 0, \theta_{+} \neq \theta_{-}$for all times. This is also a consequence of the fact that the equilibrium problem, $\partial_{x}^{3} h-w_{1} \partial_{x} h+w_{2}=0$ subject to (2.7), has no solution as it is defined with too many conditions (overdetermined).

In this rather idealized setting, it is found that after some initial transient the droplet eventually slides along the inclined plane with a constant speed. It is therefore of interest to obtain the constant speed of descent, $v_{f}$. From the original problem, this is equivalent to solving the nonlinear differential equation

$$
h(h+\lambda)\left(\partial_{x}^{3} h-w_{1} \partial_{x} h+w_{2}\right)=v_{f}
$$


with boundary conditions (2.7). Since the problem is translationally invariant we set $b_{-}=0$, so that $b_{+}=2 d$. However, the corresponding IDEs (3.26) are not amenable to further analysis, unless we consider the limit $w_{2} \ll 1$. For a steadily translating droplet so that $\dot{d}=0$, we find that the droplet radius may be written as $d=d_{0}+O\left(w_{2}^{2}\right)$, where $d_{0}$ is given by

$$
d_{0} \sqrt{w_{1}} \operatorname{coth} d_{0} \sqrt{w_{1}}=1+w_{1} / 2,
$$

which is equivalent to the equilibrium droplet radius on a horizontal substrate corresponding to the effective Bond number $w_{1}$. Using (4.2) and after some algebra, we find that

$$
\begin{aligned}
v_{f}= & \frac{\dot{b}_{+}+\dot{b}_{-}}{2}=\frac{w_{2}}{2 \ln \left(2 d_{0} / \lambda\right)+\int_{-1}^{+1}\left(\sqrt{k} \sinh \sqrt{k} /(\cosh \sqrt{k}-\cosh y \sqrt{k})+2 /\left(y^{2}-1\right)\right) \mathrm{d} y} \\
& +O\left(w_{2}^{2}\right)=\frac{w_{2}}{2 \ln \left[\left(2 / \lambda \sqrt{w_{1}}\right) \sinh \left(d_{0} \sqrt{w_{1}}\right)\right]}+O\left(w_{2}^{2}\right) .
\end{aligned}
$$

Note that $w_{1}$ does not have to be small for (4.3) to be valid. However, one has to solve the nonlinear equation (4.2) to obtain $d_{0}$. If we further assume that $w_{1} \ll 1$, we find that $d_{0}=\sqrt{3 / 2}\left[1+w_{1} / 20+O\left(w_{2}^{2}\right)\right]$, which simplifies (4.3) to

$$
v_{f}=\frac{w_{2}}{\ln \left(6 / \lambda^{2}\right)}+O\left(w_{1} w_{2}\right) .
$$

Figure 2 shows computations with ideal substrates, in which we fix $\alpha_{s}=10^{\circ}$, $\lambda=0.002$ and compare the solution with that of the nonlinear problem (4.1), with the results of our asymptotic analysis (4.3), together with its fully linearized form (4.4). In figure 2(a) we plot $v_{f}$ as a function of $w_{2}$ by fixing $\phi$ and varying $B o$ so that $0 \leqslant w_{2} \leqslant 1$. For small $\phi$ (e.g. for the $\phi=1,2$ and $5^{\circ}$ curves) the agreement of the numerics with (4.3) is excellent up to $w_{2} \approx 0.5$, whereas the linearized equation has a fair agreement up to $w_{2} \approx 0.25$. In figure $2(b)$ we show the dependence of the speed of descent on the angle of inclination when $B o$ and $\alpha_{s}$ are fixed. Equations (4.3) and (4.4) are nearly indistinguishable and are able to predict accurately the small- $\phi$ speeds. Note also that the non-monotonic dependence of $v_{f}$ on $\phi$ exhibited in figure $2(b)$ is consistent with the behaviour observed in simulations with precursor film models (see, for example, Thiele et al. 2002, figure 13(b)). In figure 2(c) we show a set of the terminal shapes attained by the droplet in its descent along the inclined plane for fixed $B o$ and $\alpha_{s}$ and different $\phi$. As $\phi$ increases the droplet wets the substrate more, developing a bulge at the front and becoming flattened at the rear. At the same time, the agreement of the matched asymptotics (see, e.g., the dashed curves in figure $2(c)$, which correspond to the leading-order outer solution) with the numerical solution to (4.1) (the solid curves in figure $2 c$ ) deteriorates. In fact, for sufficiently large inclination angles, the asymptotic analysis in $\S 3$ yields non-physical results, since the droplet thickness predicted by (3.5) is not everywhere positive, as is the case for the profile shown in figure $2(c)$, for which $\phi=30^{\circ}$. As mentioned previously, this regime requires a separate treatment, dividing further the outer region into capillarity- and gravity-dominated regions. However, once again, this is beyond the scope of the present study and we focus only on profiles that are not flattened by gravity to such an extent. 


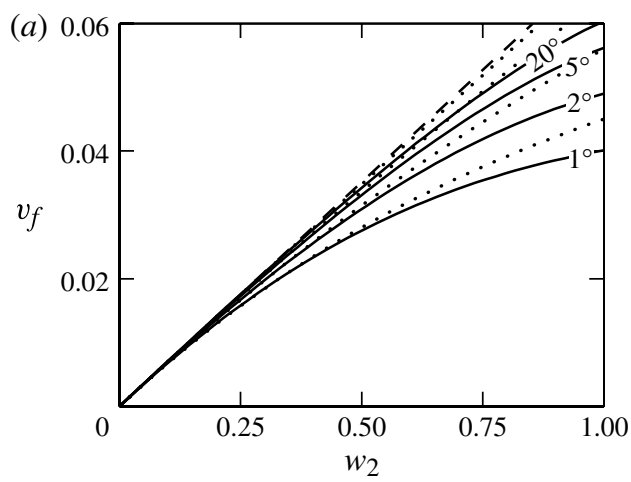

(b)

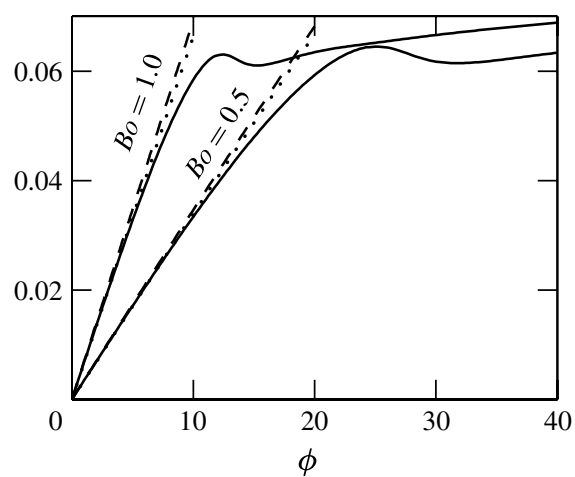

(c)

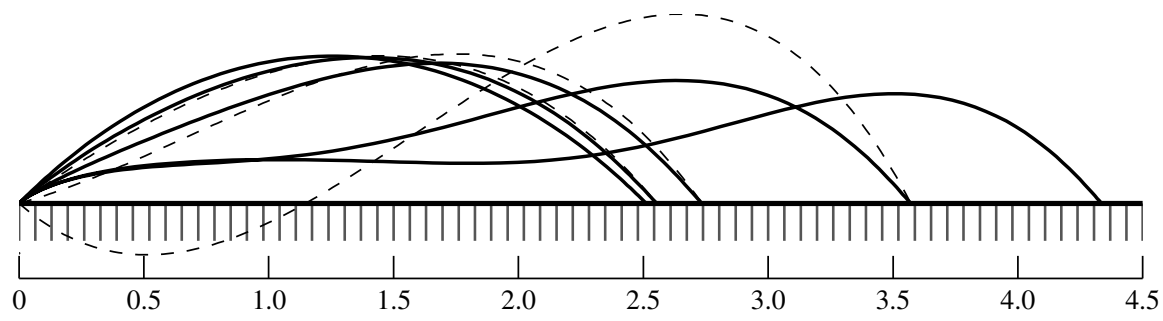

FIGURE 2. Droplets on inclined ideal substrates comparing the solutions to (4.1) (solid curves), (4.3) (dotted curves) and its linearized form (4.4) (dashed curves) for $\alpha_{s}=10^{\circ}$ and $\lambda=2 \times 10^{-3}$. (a) Plot of $v_{f}$ as a function of $w_{2}$ for $\phi=1,2,5$ and $20^{\circ}$. (b) Plot of $v_{f}$ as a function of $\phi$ for $B o=0.5$ and 1.0. (c) Droplet profiles become more elongated as $\phi$ increases, shown for $B o=0.5$ and $\phi=0,10,20,30$ and $40^{\circ}$ (solid curves); the dashed curves are the corresponding leading-order outer solutions (3.5). When $\phi=30^{\circ}$ and for the film-like solution when $\phi=40^{\circ}$, the analysis of $\S 3$ is inapplicable, since (3.5) allows for the droplet thickness to become negative, which is non-physical.

\section{Dynamics on heterogeneous substrates}

Substrate heterogeneities, chemical and/or topographical, break the translational invariance of the droplet equilibria on horizontal substrates. For inclined substrates, they can allow for the droplet to reach some equilibrium in the long-time limit, provided that the energy barriers they introduce are strong enough to oppose the downhill gravitational force. To illustrate this, we consider the spreading of a droplet on a heterogeneous substrate inclined by $\phi=15^{\circ}$, with $s(x)=0.03 \sin 20 x$ and $f(x)=1-0.1 x$. Here we have the competition of substrate topography against the body force and the chemical gradient which favour the downhill droplet motion. We note that the chemical gradient makes sense only in a limited portion of the substrate, so that $f(x)>0$ and $f(x)=O(1)$. For the other parameters, we fixed $\lambda=10^{-4}, B o=0.8$ and $\alpha_{s}=15^{\circ}$. Assuming that initially $b_{ \pm}= \pm 0.8$ and that the droplet shape in the bulk matches that of the leading-order outer solution (3.5), we solve numerically the governing partial differential equation (PDE) (2.5), subject to (2.7), using the methodology outlined in the appendix of the study by Savva \& Kalliadasis (2009), which is based on a highly accurate pseudospectral collocation scheme. For comparison, we have also solved the IDEs obtained from matching (3.26) with a Runge-Kutta scheme for time stepping, evaluating the integrals using the Legendre-Gauss quadrature (Abramowitz \& Stegun 1972, §25.4). 

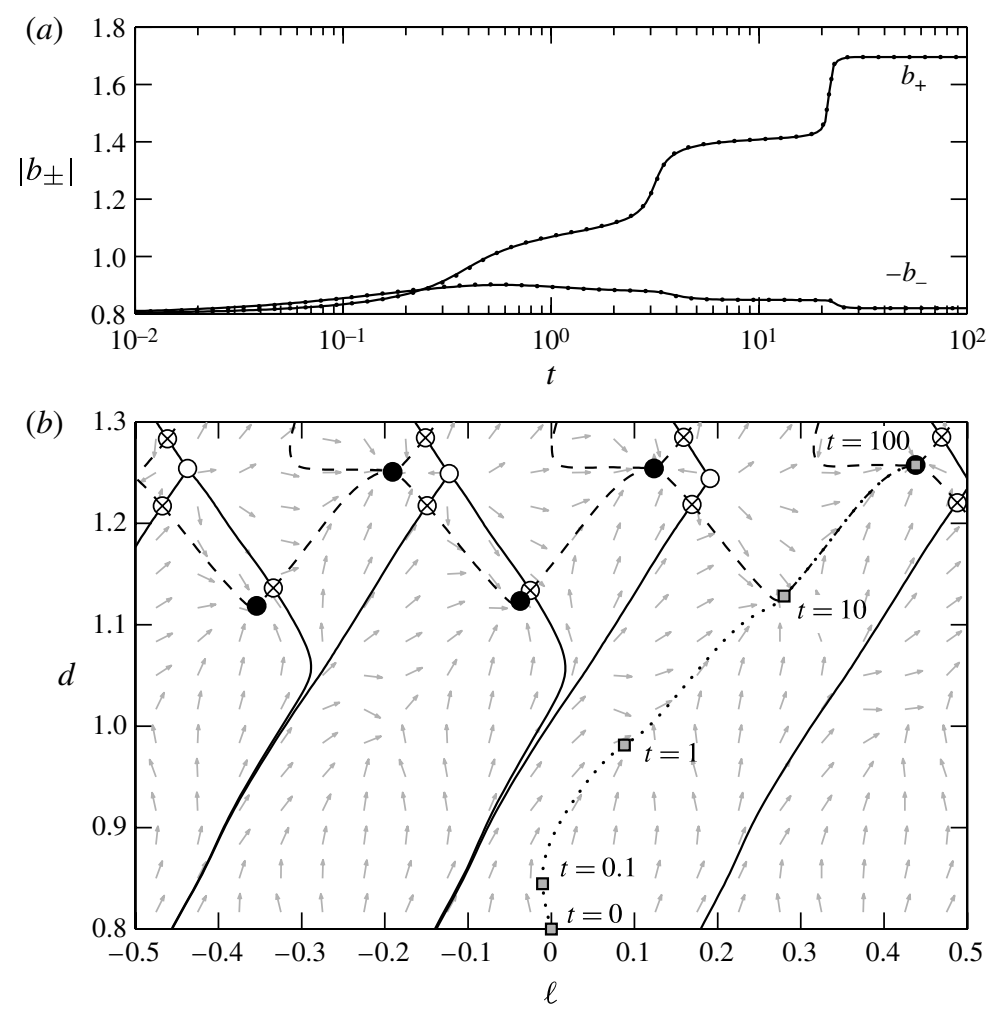

(c)

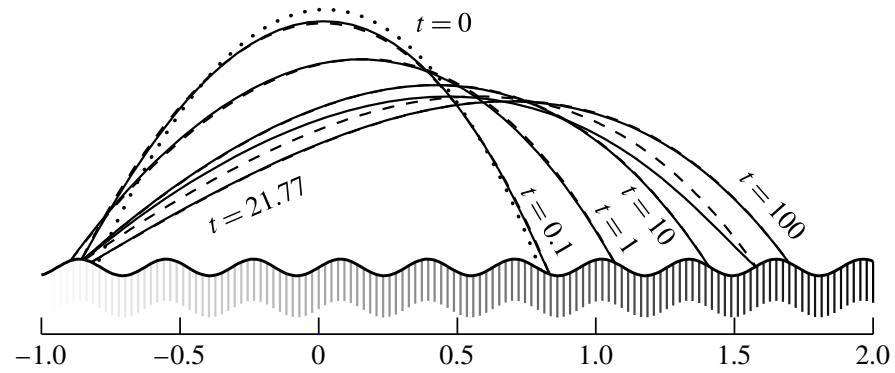

FIGURE 3. Droplet spreading on a heterogeneous substrate with $s(x)=0.03 \sin 20 x, f(x)=$ $1-0.1 x$ and $\lambda=10^{-4}$, when $B o=0.8$ and $\phi=\alpha_{s}=15^{\circ}$. (a) Time evolution of the droplet fronts when $b_{ \pm}(0)= \pm 0.8$. The solutions to the PDE (2.5) (solid curves) and the IDEs (3.26) (dotted curves) are nearly indistinguishable. (b) A snapshot of the $\ell-d$ phase plane corresponding to (3.26) showing the different equilibria, namely stable (O) and unstable nodes $(\bigcirc)$ and saddle points $(\otimes)$, whose stable and unstable manifolds are shown by the solid and dashed curves, respectively. The dotted curve is the trajectory when $b_{ \pm}(0)= \pm 0.8$, which is marked with squares at times $t=0,0.1,1,10$ and 100. (c) The shapes of the free surface at times $t=0$ (dotted curve) and $t=0.1,1,10,21.77$ and 100. The solid and dashed curves correspond to the solution of (2.5) and the leading-order outer solution (3.5), respectively. The hatches below the substrate are coloured to illustrate the chemical gradient as done in figure 1 .

In figure $3(a)$ we show the time evolution of $b_{ \pm}$, noting the excellent agreement between the solutions to the governing PDE and the IDEs obtained by matching, which can also be regarded as a strong indication of the validity of our matching 
procedure. Here we observe that the left contact point moves very little compared with the right contact point, which exhibits a series of stick-slip events as it advances through the hills and valleys of the topography. The surprisingly excellent agreement of the leading-order dynamics with the full numerical results has also been observed for horizontal heterogeneous substrates (see, e.g., Savva \& Kalliadasis 2009; Vellingiri et al. 2011). However, as pointed by Savva \& Kalliadasis (2011), there can exist initial conditions where this agreement can deteriorate, especially near the boundaries of the basins of attraction corresponding to two neighbouring stable equilibria. It is thus possible for the IDEs to yield markedly different dynamics from the governing PDE with the IDEs predicting a different final equilibrium from the one attained by the PDE. Even though this might appear to somewhat limit the predictive capabilities of (3.26), the fact that we are dealing with a much simpler, 2D dynamical system, is a significant advantage over the full PDE. More importantly, through the IDEs we can visualize the dynamics on the phase plane, which can potentially yield more insights compared with solving the PDE by a brute-force numerical approach.

Figure $3(b)$ shows a portion of the $\ell-d$ phase plane for the parameters considered above, where

$$
\ell=\frac{b_{+}+b_{-}}{2} \quad \text { and } \quad d=\frac{b_{+}-b_{-}}{2}
$$

correspond to the droplet midpoint and half-width, respectively. The choice of these variables instead of $b_{ \pm}$to visualize the phase plane was made based on the fact that $\ell$ and $d$ are more intuitive variables, showing clearly the direction of motion of the droplet and how its footprint is affected by heterogeneities. Note that only part of the phase plane makes physical sense. For large $d$ the analysis is invalidated since (3.5) may touch or intersect the substrate profile: for large $\ell, f(x)$ can become negative (for $\ell \gg 0$ ) or too large (for $\ell \ll 0$ ), violating our requirement that $f(x)$ must be strictly positive and $O(1)$. A significant advantage of a phase-plane analysis is that we can immediately identify the different equilibria and determine under what conditions these may be attained. The dotted curve shows the trajectory on the phase plane corresponding to the example considered in figure $3(a)$, for which $\ell(0)=0$ and $d(0)=0.8$. Interestingly, the largest portion of the trajectory is traversed during the early stages of the motion; the stick-slip observed at later times occurs due to the proximity of the trajectory to the unstable manifold of the saddle point, which drives the droplet to an equilibrium state. Physically, this occurs during the upward motion of the right contact point over a topographical feature. This may be inferred from figure $3(c)$, where we show snapshots of the droplet free surface at different times. The near-perfect match of the leading-order outer solution (3.5), with the solution to the PDE, is an affirmation that the dynamics is indeed quasi-static, except perhaps during the stick-slip event occurring for $10 \leqslant t \leqslant 100$. However, its duration is too brief to disrupt the otherwise excellent agreement observed in figure $3(a)$.

Clearly, heterogeneities generally tend to oppose the downhill motion of the droplet due to the energy barriers introduced. However, when there exists some asymmetry in the way heterogeneities vary in space, they can assist the downhill motion in some cases, as it occurs when the droplet moves in the presence of a favourable chemical gradient. When the chemical gradient favours the uphill motion a number of observations are worth mentioning. These are better discussed with the help of figure 4, where we consider the motion of a droplet with $B o=0.5, \alpha_{s}=10^{\circ}$ on a smooth, chemically heterogeneous substrate at various inclination angles, with $\lambda=10^{-4}, s(x)=0$ and $f(x)=1+0.1 x$. The substrate becomes progressively less 

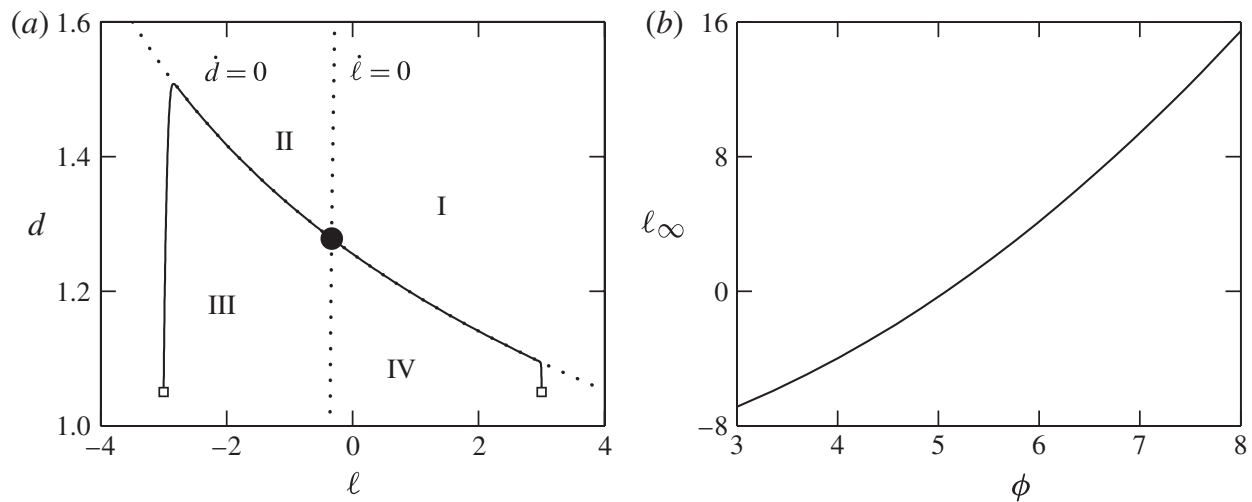

FIgURE 4. Droplets on inclined substrates with a chemical gradient, using $B o=0.5$, $\alpha_{s}=10^{\circ}, \lambda=10^{-4}, s(x)=0$ and $f(x)=1+0.1 x$. (a) Snapshot of the $\ell-d$ phase plane when $\phi=5^{\circ}$ showing the unique stable node (filled circle) and its corresponding nullclines (dotted curves), which divide the phase plane into four regions that describe different droplet behaviours. The solid curves correspond to two sample trajectories originating from $\ell(0)= \pm 3$ and $d=1.05$ (open squares). (b) Plot of the droplet midpoint at equilibrium, $\ell_{\infty}$, as a function of the inclination angle, $\phi$.

hydrophilic as we move downhill and unless the values of $x$ do not predict a non-physical value for the contact angle (e.g. negative or too large; see the earlier discussion), a stable equilibrium always exists.

In figure $4(a)$ we present a snapshot of the $\ell-d$ phase plane when $\phi=5^{\circ}$, together with sample trajectories when $\ell(0)= \pm 3$ and $d(0)=1.05$. The nullclines corresponding to the stable node, i.e. the curves for which $\dot{\ell}=0$ and $\dot{d}=0$, divide the phase plane into four regions. Using figure $4(a)$ as a reference plot, for all initial conditions in region II the droplet contracts and slides downhill, whereas in region IV it spreads and moves uphill. A droplet starting from region I moves uphill while contracting in the early stages and spreading in the final stages and in region III droplet spreading is followed by contraction, while it moves downhill. Hence, whether we observe an uphill motion or not is strongly dependent on where the droplet is located initially. Hence, if an analogy between 2D and 3D geometries can be drawn, the observations of Chaudhury \& Whitesides (1992) were likely to be made for droplets starting in regions I and IV in the phase plane in which a droplet is able to move against gravity. One can more objectively quantify whether uphill or downhill motion occurs, by tracking the location of the stable equilibrium in relation to initial conditions which are symmetric relative to the origin, i.e. $\ell(0)=0$. For the parameters considered above, the $\ell$ coordinate of the stable node, $\ell_{\infty}$, crosses the origin when $\phi \approx 5.08^{\circ}$. As the inclination angle is further increased the droplet attains an equilibrium that is located further downhill (see figure $4 b$ ).

When we consider the effects of both types of heterogeneities, it is generally more difficult even to qualitatively predict the droplet behaviour. For example, figure 5 depicts the motion of a droplet on a heterogeneous substrate with $s(x)=0.03$ sin $10 x$, $\lambda=10^{-4}$, when $B o=0.5, \alpha_{s}=\phi=15^{\circ}$ and $b_{ \pm}(0)= \pm 0.8$ for different heterogeneity functions. When the substrate is chemically homogeneous, the substrate asperities will prevent the droplet from flowing downhill. On the other hand, when the combined effects of this single-wavelength topography with a single-wavelength chemical 

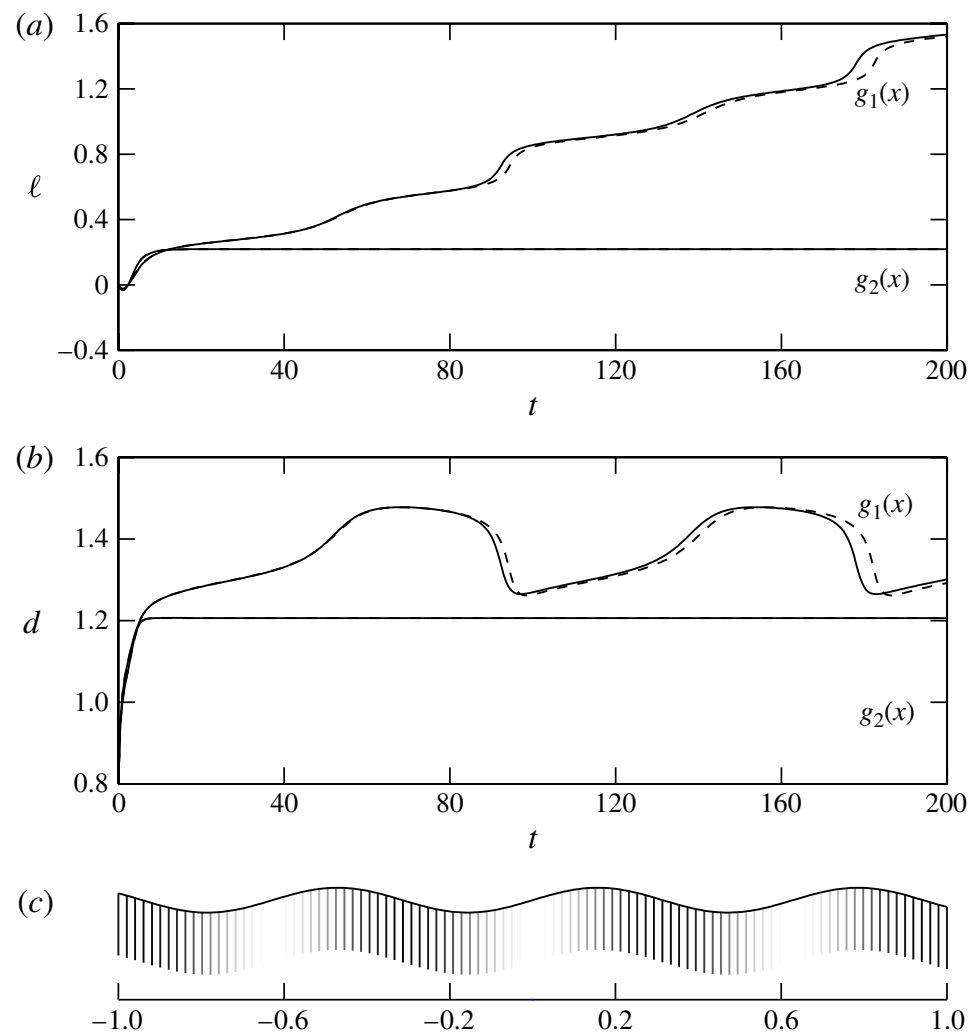

FIGURE 5. Evolution of the droplet midpoint $(a)$ and droplet half-width $(b)$ for a droplet on a heterogeneous substrate with $s(x)=0.03 \sin 10 x, \lambda=10^{-4}$, when $B o=0.5, \alpha_{s}=\phi=15^{\circ}$ and $b_{ \pm}(0)= \pm 0.8$ for two different chemical heterogeneity functions, $f_{1}(x)=1+0.2 \cos 10 x$ and $f_{2}(x)=1+0.2 \sin 10 x$. For $f_{1}(x)$, the droplet moves downhill whereas for $f_{2}(x)$ the droplet is eventually pinned due to the heterogeneities. The solutions to the PDE (2.5) (solid curves) and the IDEs (3.26) (dashed curves) are indistinguishable for $f_{2}(x)$, whereas their relative difference for $f_{1}(x)$ is less than $10 \%$ for $t \approx 180$. (c) A configuration favouring the downhill motion for single-harmonic heterogeneities: the peaks in the topography are located half a wavelength to the right of the least wetting regions.

heterogeneity are considered, the picture can change drastically. We performed numerical experiments for heterogeneity functions of the form $f(x)=1+\epsilon \sin m x$ for $-0.5 \leqslant \epsilon \leqslant 0.5$ and $m$ being an integer chosen between 1 and 30 , and in all cases the droplet was eventually pinned by the heterogeneities. Similarly, for $f(x)=1+\epsilon \cos m x$, pinning is also observed for this range of values of $\epsilon$ and $m$ unless $m=10$ and $\epsilon$ ranges approximately between 0.138 and 0.345 . In figure $5(a, b)$ we show two examples of different chemical heterogeneity functions, $f_{1}(x)=1+0.2 \cos 10 x$ and $f_{2}(x)=1+0.2 \sin 10 x$. For $f_{1}(x)$ the droplet eventually moves downhill, whereas for $f_{2}(x)$ the droplet reaches an equilibrium.

Hence, from our numerical experiments it appears that to optimize the downhill droplet motion, the wavelengths of the two heterogeneity types have to be matched and the peaks in the heterogeneity functions must be out of phase, with the peaks in the topography situated half a wavelength to the right of the peaks in the chemical heterogeneities (see figure $5 c$ ). In this configuration, the droplet will gain momentum 
as the contact line moves down a topographical hill, which is further assisted by the smaller local contact angles. If $\epsilon$ is neither too high nor too low, this momentum will be enough for the droplet to overcome the energy barrier during the uphill phase of the contact line motion. For any other configuration, the uphill motion appears to be impeded. Naturally, however, the strength of both types of heterogeneities must be sufficiently low for this type of motion to be observed. Moreover, as we shall see in $\S 6$, even though in general the $\pi / 2$ phase difference between the heterogeneities yields reduced critical inclination angles compared to in-phase configurations, we cannot generally assert that a phase difference of exactly $\pi / 2$ is indeed the optimum configuration.

In the simulation for $f_{1}(x)$, noteworthy is the comparatively poor agreement between the solution to the PDE and the equations obtained by matching, but as mentioned previously, such discrepancies are to be expected, particularly for larger Bond numbers and inclination angles for which the droplet can more easily overcome the heterogeneity barriers. Nevertheless, in all our simulations, the difference between the solution to the governing PDE and the solution to the system of IDEs obtained by matching did not appear to grow significantly over the duration of the simulations and typically remained below $10 \%$, which may be attributed, at least in part, to the periodicity of the heterogeneities considered.

\section{Critical inclination angle}

The main focus of many experiments reported in the literature is the determination of $\phi_{\text {crit }}$ and how it is affected by different factors. As mentioned in $\S 4$, our model suggests that homogeneous substrates cannot possibly pin the droplet, from which one can infer that substrate heterogeneities is the principal source for contact angle hysteresis, a view held by the majority of the researchers in the field.

From the preceding discussion, we see that, indeed, if heterogeneities are sufficiently strong, the droplet can be pinned on the substrate. Hence, it is of interest to study the droplet behaviour as the inclination angle is varied. In figure 6 , we show the results of such a calculation, for a substrate with $s(x)=0.02 \sin 10 x, f(x)=1+0.3 \sin 20 x$ and $\lambda=10^{-4}$, when $B o=0.8, \theta=15^{\circ}$ and $b_{ \pm}(0)= \pm 1.1$. From figure $6(a)$, where we plot the evolution of the droplet midpoint, $\ell$, we readily deduce from the three inclination angles considered that $\phi_{c r i t}$ must lie between 14 and $15^{\circ}$. However, in an experimental setting, $\phi_{c r i t}$ may be underestimated. This is because $\phi=15^{\circ}$ might be perceived to be below $\phi_{c r i t}$, due to the fact that the droplet stays pinned at the heterogeneities for a relatively long time, which is significantly longer than the typical time it takes for a droplet to spread on a horizontal substrate. Hence, a systematic discrepancy is expected between the experimental measurements of $\phi_{\text {crit }}$ and the actual value. However, this discrepancy will most likely be small and within the accuracy limits of a measuring instrument.

It is also interesting to note the similarity in the droplet behaviour observed for $\phi=15^{\circ}$ and $\phi=16^{\circ}$, where we see that the two motions exhibit identical characteristics but at different times. For example, the steps in $\ell$, which are separated by the wavelength of the substrate heterogeneities, $\pi / 5$, occur at significantly longer times for $\phi=15^{\circ}$ compared with $\phi=16^{\circ}$ (see figure $6 a$ ). The same can be said for the relaxation-type oscillations observed for the evolution of the half-width of the droplet (see figure $6 b$ ). While there is some similarity in the actual curves, the highly nonlinear nature of the equations, both the PDE and those obtained by matching, 
(a)

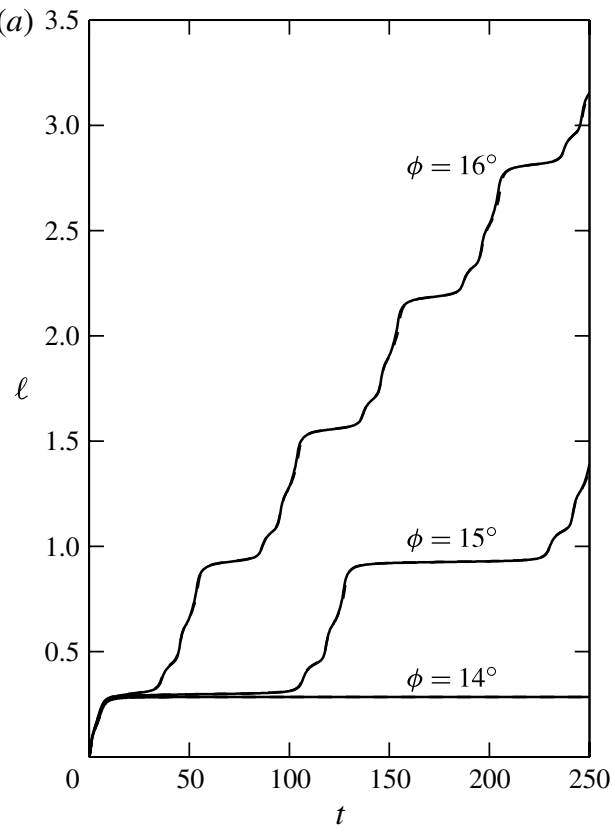

(b)
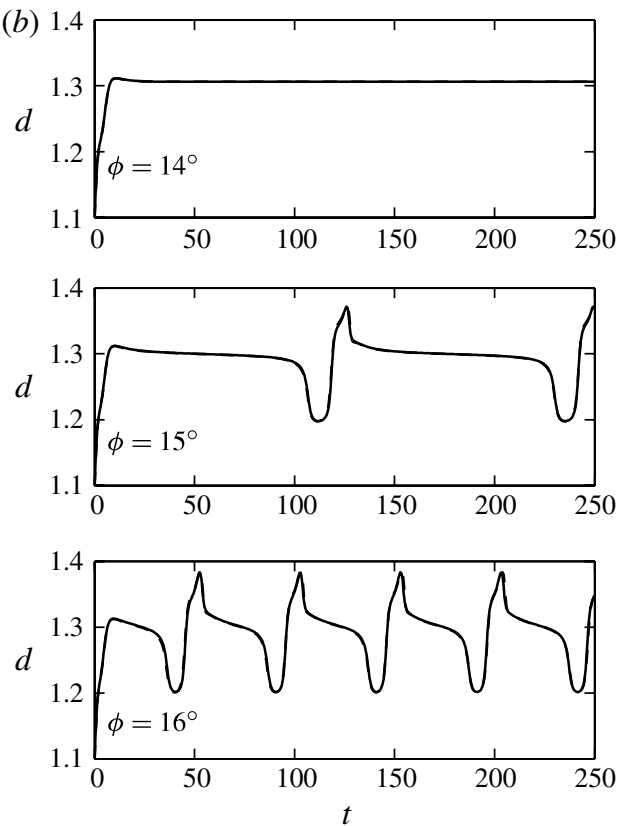

FIGURE 6. Evolution of the droplet midpoint $(a)$ and droplet half-width $(b)$ at various inclination angles, $\phi$, for a droplet on a heterogeneous substrate with $s(x)=0.02 \sin 10 x$, $f(x)=1+0.3 \sin 20 x$ and $\lambda=10^{-4}$, when $B o=0.8, \alpha_{s}=15^{\circ}$ and $b_{ \pm}(0)= \pm 1.1$. For lower $\phi$ the droplet is pinned at the heterogeneities; above some critical angle it moves downhill with average speeds that increase as the angle is increased. When $\phi=15^{\circ}$, just above the critical inclination angle, the droplet is temporarily pinned for relatively long time intervals. The solutions to the PDE (2.5) (solid curves) and the IDEs (3.26) (dashed curves) are visually indistinguishable.

preclude any further analysis in this direction, given also that the observed stretching in time was found to be non-uniform.

Clearly, as the inclination angle changes, some type of bifurcation is expected to occur. By investigating the equations obtained from matching (3.26) in a $2 \mathrm{D}$ phase plane, the bifurcation events can become more transparent. It is therefore of interest to observe the topological changes to the $\ell-d$ phase plane as the inclination angle is varied. For simplicity, we will only consider the case of topographical heterogeneities, since the same observations are expected for a chemically heterogeneous substrate and a substrate with both types of heterogeneities. For the sake of illustration, we will also assume a single harmonic for the topography, even though more complicated forms could be considered as well (e.g. substrates with random topography; see Savva, Kalliadasis \& Pavliotis 2010; Savva, Pavliotis \& Kalliadasis 2011a,b).

In figure 7 we show snapshots of the $\ell-d$ phase plane as $\phi$ increases for a substrate with $f(x)=1, s(x)=10^{-3} \cos 50 \pi x$ and $\lambda=10^{-4}$ with $B o=0.4$ and $\alpha_{s}=12^{\circ}$. In order to better demonstrate the influence of a varying inclination angle, the behaviour of two droplets, marked as $A$ and $B$ in figure 7, is tracked. They are both at a stable equilibrium when $\phi=0^{\circ}$ (the two equilibria are marked with grey-filled circles in figure $7 a$ ) and their evolution is observed by inclining the substrate in increments of $\Delta \phi=0.5^{\circ}$, after which they are allowed to equilibrate to a nearby stable state. When the inclination angle changes to $\phi=2^{\circ}$, the equilibrium associated with 

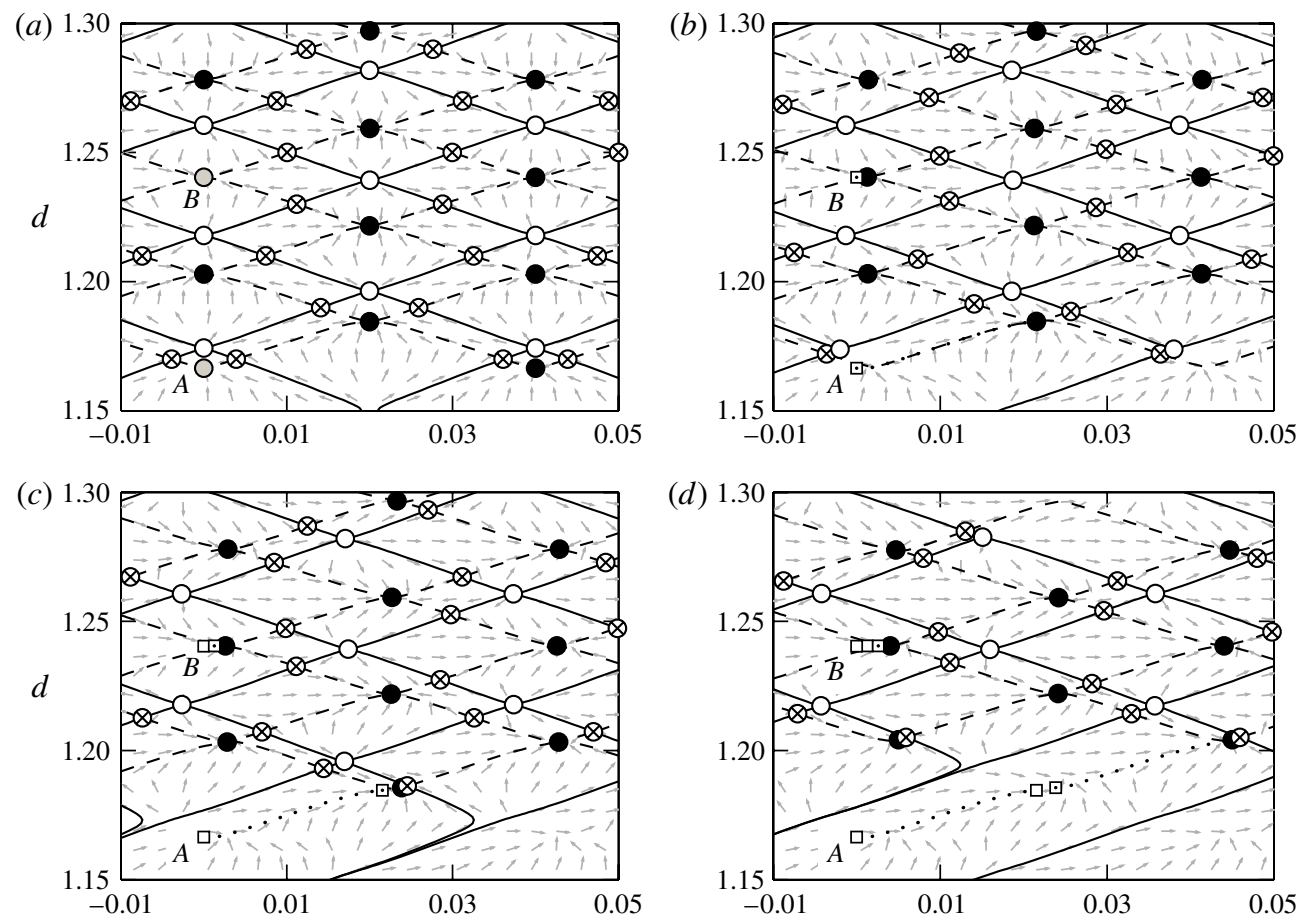

(d)
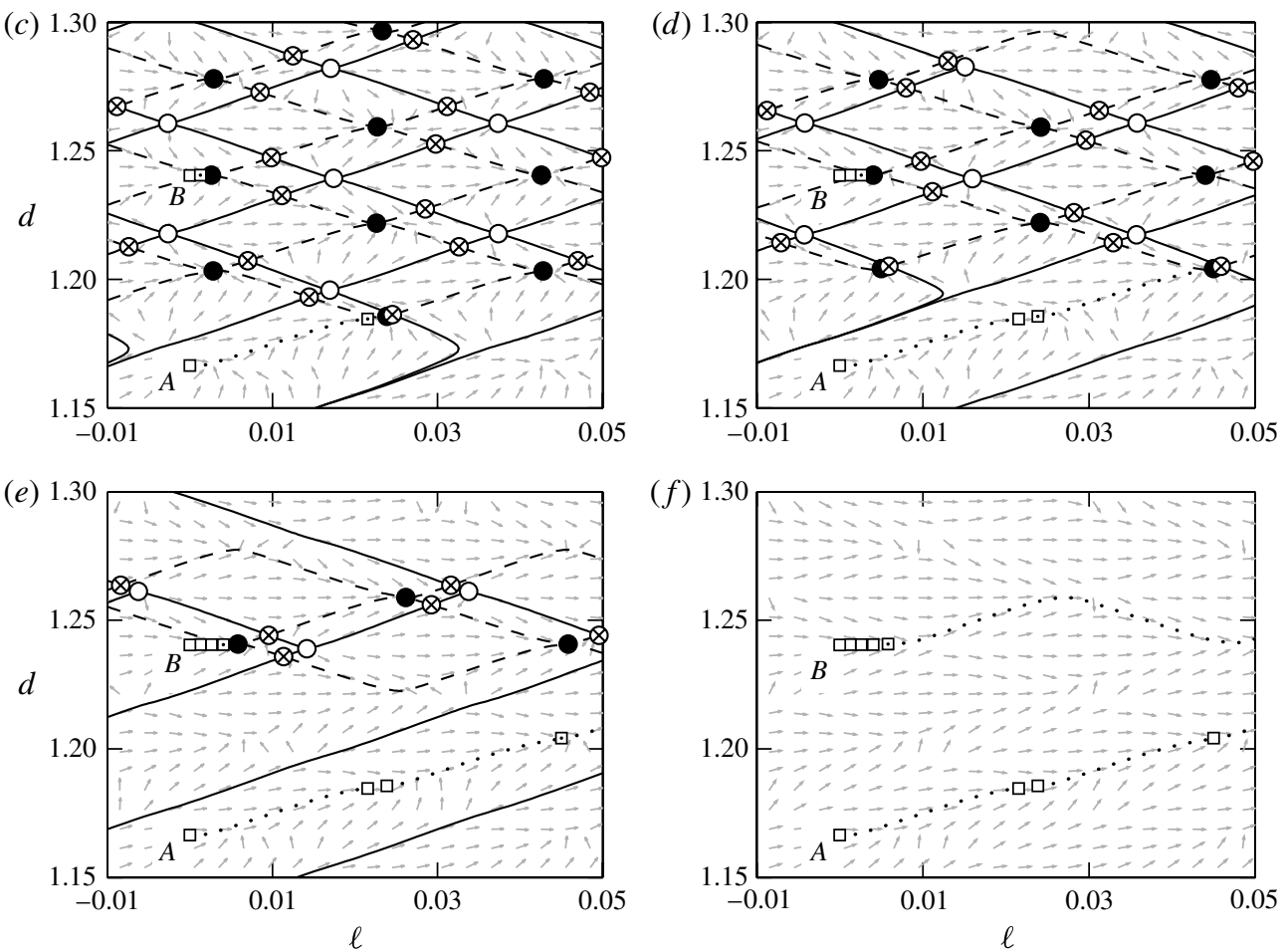

FIGURE 7. Topological changes in the $\ell-d$ phase plane as the inclination angle changes for a substrate with $f(x)=1, s(x)=10^{-3} \cos 50 \pi x$ and $\lambda=10^{-4}$ with $B o=0.4$ and $\alpha_{s}=12^{\circ}$. Plots $(a)-(f)$ correspond to $\phi=0,2,4,6,8$ and $10^{\circ}$, respectively. As $\phi$ increases, equilibria coalesce and annihilate each other until $\phi>\phi_{\text {crit }}$ in plot $(f)$, where there are no equilibria. The dotted curves show the evolution of two droplets, $A$ and $B$, which are initially at a stable equilibrium on a horizontal substrate (the grey-filled circles in $(a)$ ) and the squares mark the starting positions for each $\phi$ (for the remaining symbols and curves, refer to the caption of figure $3 b$ ). When $\phi<\phi_{\text {crit }}$, droplet $B$ appears to be pinned macroscopically, whereas droplet $A$ would briefly exhibit a stick-slip behaviour before reaching one of the nearby stable states.

droplet $A$ disappears and the droplet is displaced by a distance $\Delta \ell \approx 0.02$ downhill (see figure $7 b$ ). As the inclination angle changes further (see figure $7(c-e)$ ), we see a series of bifurcations occurring in which equilibria gradually disappear. In this process, droplet $A$ is frequently unpinned due to the annihilation of its stable 


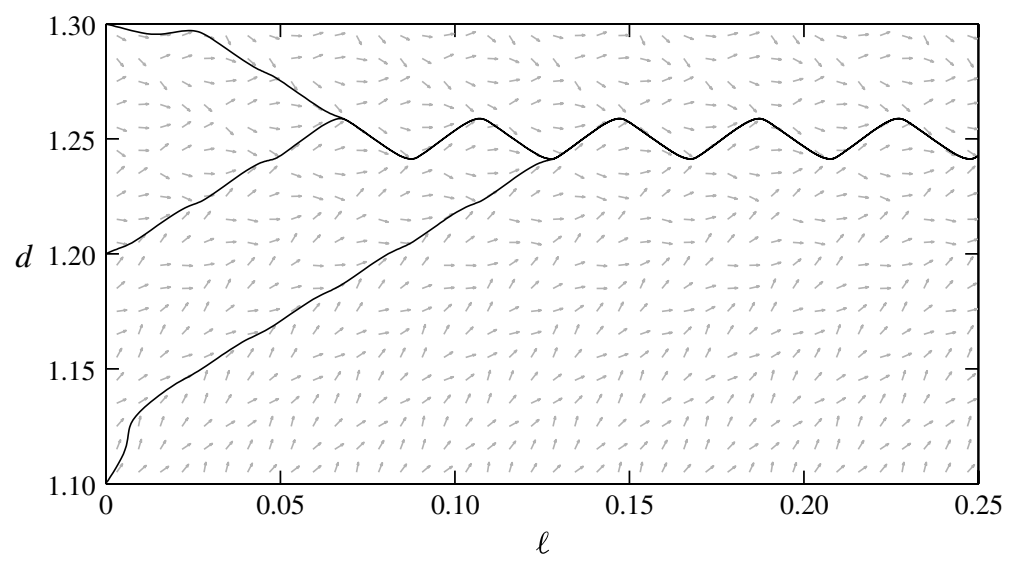

FIGURE 8. Phase plane corresponding to the substrate in figure 7 when $\phi=10^{\circ}>\phi_{\text {crit }}$ with trajectories for different initial conditions (see also figure $7 f$ ). Even though the motion is non-periodic, the droplet radius exhibits periodic oscillations in the long-time limit, regardless of the initial condition.
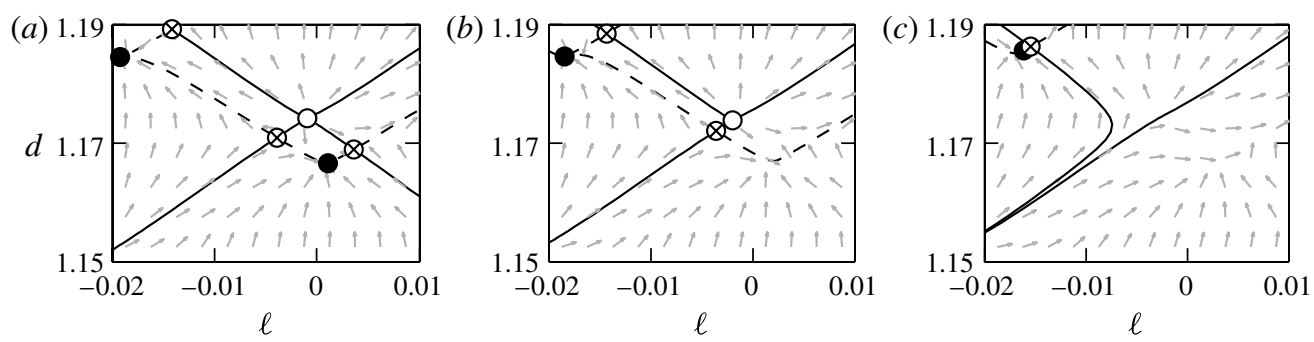

FIGURE 9. Coalescence and annihilation of fixed points as the inclination angle increases (zoomed-in portions of the phase planes in figure $6 a-c$ ): $(a) \phi=0^{\circ}$, the 'undisturbed' phase plane; (b) $\phi=2^{\circ}$, shortly after the coalescence of a stable node and a saddle point; $(c) \phi=4^{\circ}$, shortly after the coalescence of an unstable node and a saddle point.

state and dynamically seeks a nearby stable state. On the other hand, droplet $B$ moves very slightly downhill as its associated stable state is displaced to the left and macroscopically it appears to be pinned at the substrate. These behaviours are consistent with the experiments reported by Berejnov \& Thorne (2007), in which they observed a transient pinning and depinning of the contact line as the inclination angle was varied.

Figure $7(f)$ shows that when the inclination angle exceeds some critical angle, $\phi_{\text {crit }}$, all equilibria disappear and the droplets freely move downhill. In this regime, i.e. when $\phi>\phi_{\text {crit }}$, and for the periodic substrate heterogeneities considered here, the droplet half-width eventually assumes a universal periodic behaviour which is the same for all droplets of the same size, regardless of their initial state (see figure 8).

The bifurcations occurring as $\phi$ changes become clearer if we look at a zoomed-in portion of the phase plane of figure 7 (see figure 9). Starting from a horizontal substrate (figure $8 a$ ) and gradually increasing $\phi$, a saddle point approaches a stable node and upon coalescence they annihilate each other (see figure $9 b$ ). Further increasing $\phi$, an unstable node approaches a saddle point, with the two fixed points 
also coalescing and disappearing (figure $9 c$ ). This process requires that fixed points appear in pairs, where stable and unstable nodes merge with nearby saddle points. In cases where an odd number of fixed points exist when $\phi=0^{\circ}$ (e.g. for a single-trench or single-ridge topography, as shown in figures 13 and 14 in Savva \& Kalliadasis 2009), additional equilibria are created for $\phi$ slightly different from $0^{\circ}$, so that the mechanism described above can take place. Note, however, that in other substrate profiles such as the ratchet profiles considered later on, it is possible to observe the emergence of new equilibria for certain values of $\phi$, which nevertheless eventually coalesce and disappear in the manner described above.

The critical inclination angle, $\phi_{\text {crit }}$, can be determined relatively easily numerically but a fully analytical prediction is impossible. Analytical estimates can be obtained with a number of simplifying assumptions in the limit of weak and slowly-varying heterogeneities. Starting from the requirements for equilibrium, namely

$$
\theta_{ \pm}=\alpha_{ \pm}=f\left(b_{ \pm}\right)+O\left(\tan ^{2} \alpha_{s}\right)
$$

we seek the largest value of $\phi$ allowed by the solutions to the system (6.1). We further assume small-amplitude topographical features, so that we may take contributions due to $s(x)$ to be of higher-order compared with contributions due to $s^{\prime}(x) \ll 1$. We also assume small deviations from a chemically homogeneous substrate, by writing $f(x)=1+g(x)$ with $g(x) \ll 1$. By doing so, the system (6.1) can be written as

$$
\frac{k^{2}}{2 d^{2}(k \operatorname{coth} k-1)} \pm \frac{w_{2} d^{2}}{k^{2}}(k \operatorname{coth} k-1) \pm s_{ \pm}^{\prime}=1+g\left(b_{ \pm}\right)+O\left(\tan ^{2} \alpha_{s}, s_{ \pm}\right) .
$$

By considering the sum of (6.2) we get

$$
\frac{k^{2}}{d^{2}(k \operatorname{coth} k-1)}=2+g_{+}+g_{-}+s_{-}^{\prime}-s_{+}^{\prime}+O\left(\tan ^{2} \alpha_{s}, s_{ \pm}\right),
$$

where we set $f_{ \pm}=f\left(b_{ \pm}\right)$. On the other hand, the difference of (6.2) yields

$$
w_{2}=\frac{1}{2}\left(2+g_{+}+g_{-}+s_{-}^{\prime}-s_{+}^{\prime}\right)\left(g_{+}-g_{-}-s_{+}^{\prime}-s_{-}^{\prime}\right)+O\left(\tan ^{2} \alpha_{s}, s_{ \pm}\right) .
$$

To make analytical progress, (6.3) and (6.4) need to be simplified further, by retaining only the linear terms in $g_{ \pm}$and $s_{ \pm}^{\prime}$ in (6.4). Furthermore, note that the left-hand side of (6.3) equals two for a droplet at rest on an ideal and horizontal substrate and make the a priori assumption that its corrections are of higher order compared with $O\left(g_{ \pm}\right)$and $O\left(s_{ \pm}^{\prime}\right)$ terms. As we shall soon see, however, this is a drastic step that does not always yield a satisfactory estimate.

All of these assumptions lead to a considerably simpler system and the problem of estimating $\phi_{\text {crit }}$ can be cast as a maximization problem, in which we need to find the maximum of

$$
w_{2}=g_{+}-g_{-}-s_{+}^{\prime}-s_{-}^{\prime},
$$

given

$$
g_{+}+g_{-}+s_{-}^{\prime}-s_{+}^{\prime}=0,
$$

noting that the higher-order corrections in (6.5) and (6.6) are discarded for simplicity. Despite these considerable simplifications, estimating $\phi_{c r i t}$ analytically remains rather difficult. However, if we assume single-wavelength, periodic heterogeneity functions, e.g.

$$
s(x)=s_{0} \cos m x
$$




$$
g(x)=g_{0} \cos (m x+\epsilon),
$$

where $2 \pi / m$ is the wavelength of the heterogeneities and $\epsilon$ corresponds to the phase shift between $s(x)$ and $g(x)$, analytical progress can be made. In this case, (6.5) and (6.6) become, respectively,

$$
\begin{aligned}
w_{2} & =2 s_{0} m \sin m \ell \cos m d-2 g_{0} \sin m d \sin (m \ell+\epsilon) \\
0 & =2 s_{0} m \sin m d \cos m \ell+2 g_{0} \cos m d \cos (m \ell+\epsilon)
\end{aligned}
$$

when written in terms of $d$ and $\ell$. Using (6.10) to eliminate $d$ in (6.9), we finally find

$$
w_{2}=\frac{s_{0}^{2} m^{2} \sin 2 m \ell+g_{0}^{2} \sin (2 m \ell+2 \epsilon)}{\sqrt{s_{0}^{2} m^{2} \cos ^{2} m \ell+g_{0}^{2} \cos ^{2}(m \ell+\epsilon)}} .
$$

Hence, to determine $\phi_{c r i t}$, or equivalently the critical $w_{2}$, we need to obtain the maximum of (6.11) for all values of $\ell$. Omitting the details of such a lengthy calculation, we find that

$$
w_{2} \leqslant 2 \sqrt{s_{0}^{2} m^{2}+g_{0}^{2}-\left|2 m g_{0} s_{0} \sin \epsilon\right|} .
$$

From the definition of $w_{2}$, the following estimate for $\phi_{\text {crit }}$ can be obtained

$$
\sin \phi_{c r i t} \approx \frac{2 \tan \alpha_{s} \sqrt{s_{0}^{2} m^{2}+g_{0}^{2}-2\left|m g_{0} s_{0} \sin \epsilon\right|}}{B o} .
$$

Despite the simplifying assumptions required to deduce (6.13), it is important to note that this simple formula can be used to explain qualitatively some of the observations of figure 3 and why having heterogeneities that are out of phase by $\pi / 2$ eventually results in reduced critical angles, compared with the in-phase configuration.

To test the validity of (6.13), we performed a series of numerical experiments for a variety of substrates for fixed $B o=0.4$ and $\alpha_{s}=12^{\circ}$. To determine $\phi_{\text {crit }}$, we exploited the fact that substrates under consideration are periodic and restricted our attention within a wavelength of the substrate, computing all stable equilibria when $\phi=0^{\circ}$. Using numerical continuation techniques, we determined the fate of these stable equilibria as $\phi$ increased until they disappeared.

Equation (6.13) predicts that $\phi_{c r i t}$ is approximately independent of $m$ for purely chemically heterogeneous substrates $\left(s_{0}=0, g_{0} \neq 0\right)$. Our calculations in figure $10(a)$, however, demonstrate that $\phi_{c r i t}$ becomes nearly independent of $m$ and (6.13) is in good agreement with the numerical experiments provided that $m \gg 1$, which is perhaps towards the limit of applicability of the long-wave approximation. On the other hand, (6.13) appears to be an excellent estimate for purely topographical heterogeneities $\left(s_{0} \neq 0, g_{0}=0\right)$ for all amplitudes used in our computations.

Our computations also revealed that the dependence of $\phi_{c r i t}$ on $\epsilon$ is in fact considerably more complex than that predicted by (6.13). Better agreement was typically observed when the strength of the topographical heterogeneities was lower than those of the chemical ones (see, e.g., figure 11a), but for slightly higher amplitudes in the substrate topography, $\phi_{\text {crit }}$ tends to exhibit a sawtooth behaviour as $\epsilon$ is varied, such as that shown in figure 11(b). Even though $\phi_{\text {crit }}$ appears to be minimized when $\epsilon$ is close to $\pi / 2$, the nonlinearities of the corresponding equilibrium equations preclude any further analysis. It is nevertheless interesting to note the comparatively better agreement between our numerical experiments and (6.13) when $\sin \epsilon$ is small as shown in all plots of figure 11 . 

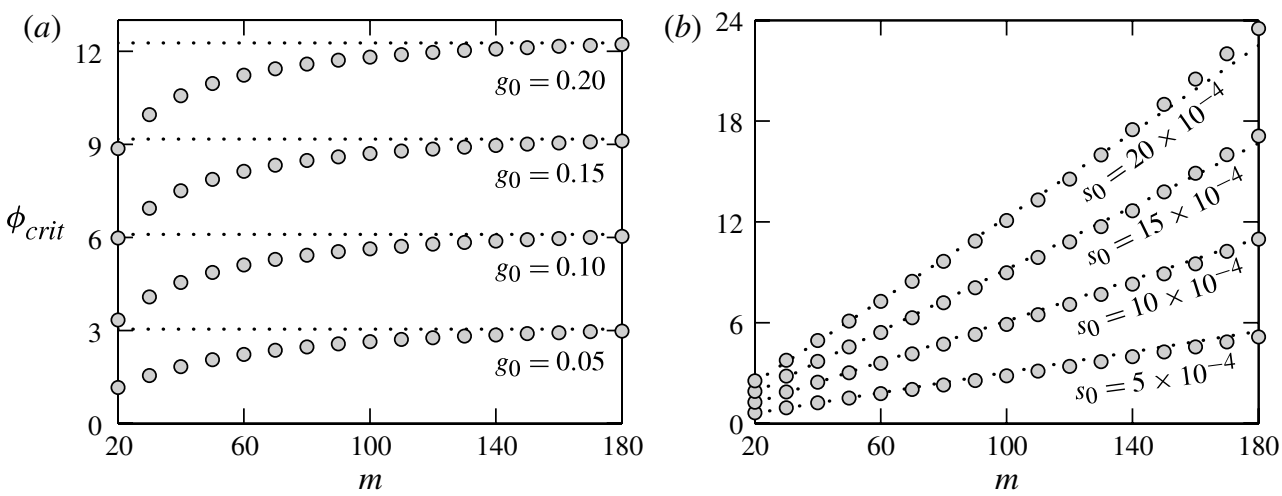

FIGURE 10. Variation of $\phi_{c r i t}$ as a function of $m$ for $(a)$ purely chemical and $(b)$ purely topographical substrates of different strengths, comparing simulation results (circles) together with the corresponding theoretical curve and equation (6.13) (dotted lines) using the heterogeneity profiles given by (6.7) and (6.8), when $B o=0.4$ and $\alpha_{s}=12^{\circ}$.
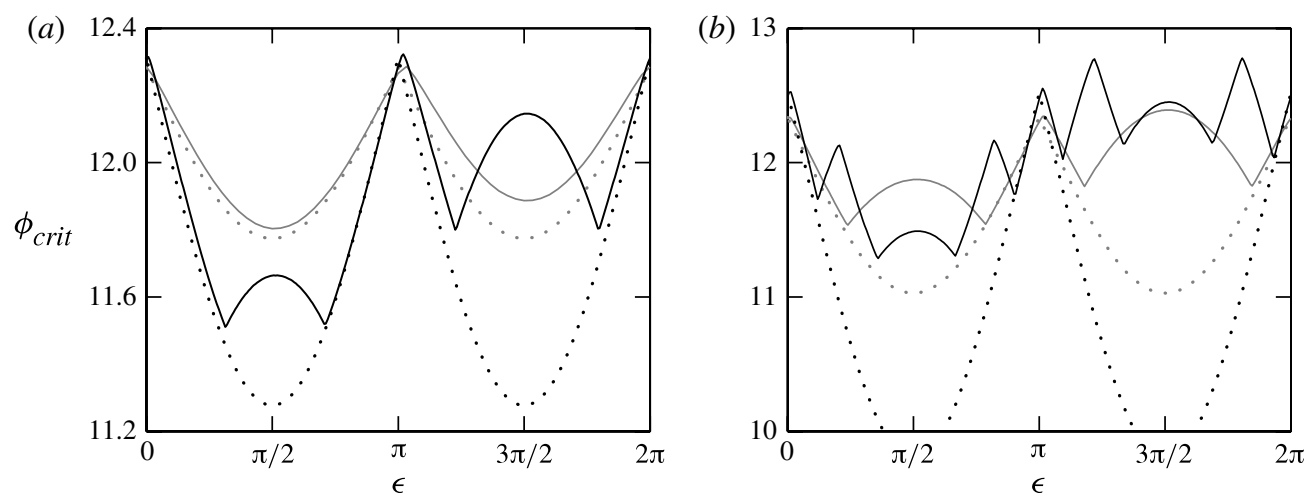

Figure 11. Plots of $\phi_{\text {crit }}$ as a function of $\epsilon$ when $B o=0.4$ and $\alpha_{s}=12^{\circ}$ to compare the theoretical result, equation (6.13) (dotted curves) and the simulation results (solid curves), using (6.7) and (6.8) to prescribe the heterogeneities with $m=200$ and $g_{0}=0.2$ : (a) $s_{0}=4 \times 10^{-5}$ (grey curves) and $s_{0}=8 \times 10^{-5}$ (black curves); $(b) s_{0}=1 \times 10^{-4}$ (grey curves) and $s_{0}=2 \times 10^{-4}$ (black curves).

Our discussion on the critical angle is concluded by noting that $\phi_{\text {crit }}$ is also strongly dependent on whether the substrate heterogeneities are symmetric or not. For example, on ratchet substrates one expects markedly different $\phi_{\text {crit }}$, depending on the orientation of the substrate in relation to the steep gradients in the heterogeneities. To demonstrate this effect, we considered a chemically homogeneous substrate and a substrate topography of the form

$$
s(x)=s_{0} c(v) \frac{\tanh \left(v \sin \frac{\kappa x}{2}\right)}{\tanh v \ln \cosh v} \ln \left[\frac{\cosh \left(v \cos \frac{\kappa x}{4}\right)}{\cosh \left(v \sin \frac{\kappa x}{4}\right)}\right],
$$

that generates controllable ratchet profiles depending on the wavenumber $\kappa$, the amplitude $s_{0}$ and the parameter $v$, going from a purely sinusoidal profile 
(a)

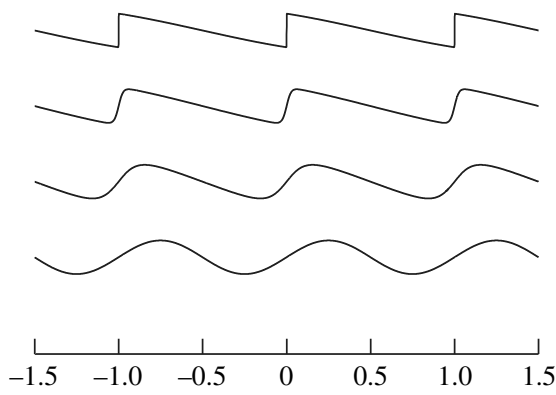

(b)

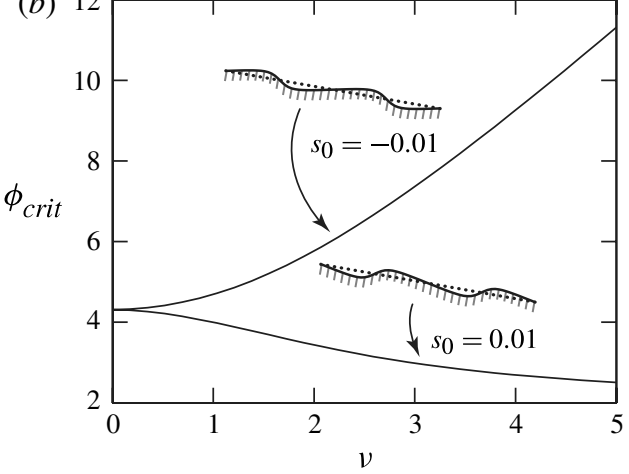

FIGURE 12. (a) Realizations of (6.14) when $s_{0}=0.1, \kappa=2 \pi$ for various $v$ (plots from bottom to top correspond to $v=0,3,12$ and 500, respectively). (b) Critical inclination angle as a function of $v$ when $B o=0.5, \alpha_{s}=12^{\circ}$ for $\kappa=12$ and $s_{0}= \pm 0.01$. Lower $\phi_{\text {crit }}$ is observed for $s_{0}=0.01$, which can be attributed to the fact that the flattened portions of the topography are inclined more steeply with respect to the horizontal in comparison to the case when $s_{0}=0.01$. Note that the vertical scale of the sketches included are exaggerated for clarity. The dotted curves in those sketches correspond to the reference plane $s_{0} \equiv 0$.

as $v \rightarrow 0\left(s(x)=s_{0} \sin \kappa x\right)$ to the sawtooth profile

$$
s(x)=s_{0} \operatorname{sign}\left(\sin \frac{\kappa x}{2}\right)\left(\left|\cos \frac{\kappa x}{4}\right|-\left|\sin \frac{\kappa x}{4}\right|\right)
$$

as $v \rightarrow \infty$ (see figure $12 a$ for a few representative cases). The function $c(v)>0$ is numerically determined to ensure that the amplitude of the profile is equal to $s_{0}$ (in the limiting cases, $v \rightarrow 0$ and $\infty, c(v)$ becomes 2 and 1 , respectively).

Figure $12(b)$ reveals how the sharpness and the orientation of the substrate can affect the critical inclination angle, when $B o=0.5, \alpha_{s}=12^{\circ}, \kappa=12$ and $\left|s_{0}\right|=0.01$. The sharpness of the features is governed by the choice of $v$, whereas the orientation of the features is determined by the sign of $s_{0}$ (see the sketches included in figure $12 b$ ). We readily see that the asymmetry in the features has a remarkable influence on the critical inclination angles. When $s_{0}=0.01$, i.e. when the features are oriented so that the droplet has to climb a step for large $v$, the critical inclination angle appears to decrease as $v$ increases. On the other hand, for $s_{0}=-0.01$, i.e. when the features are oriented so that the droplet has to go down a step for large $v, \phi_{\text {crit }}$ increases steadily as $v$ increases.

While these results appear to contradict what is observed in nature (e.g. on butterfly wings; see Zheng, Gao \& Jiang 2007) or in related experiments (see, e.g., Sheng \& Zhang 2011), where the step-down motion yields smaller $\phi_{\text {crit }}$, we note that these observations are for hydrophobic substrates, whereas our analysis focuses on the smallcontact-angle regime. More importantly, as the sketches of figure 12(b) illustrate, the nearly flat portions of the substrate topography appear to be less inclined with respect to the horizontal when $s_{0}=-0.01$. This can explain the tendency of the droplet fronts to be pinned at the steps of the topography, since the downward driving force along the flat portions is considerably reduced compared with the case when $s_{0}=0.01$. 


\section{Concluding remarks}

We have considered the motion of $2 \mathrm{D}$ droplets on inclined heterogeneous substrates in the limit of small contact angles and moderate gravity. We have attempted, for the first time, to look at the dynamic behaviour of a droplet on an inclined substrate without imposing a priori the presence of contact angle hysteresis, but rather by allowing for hysteresis to arise naturally through the substrate heterogeneities. By assuming inertia-less, quasi-static dynamics in the presence of slip, we obtained evolution equations for the location of the two moving fronts using principles from matched asymptotic analysis. The validity of our matching procedure was verified by comparisons with numerical solutions to the governing equation arising from the long-wave expansion.

We confirmed that without imposing hysteresis, droplets cannot be kept at equilibrium on ideal substrates, deducing analytically an estimate for the speed of descent, valid for small gravitational effects and inclination angles. When the substrate is heterogeneous, we confirmed that indeed the droplet can attain an equilibrium on the substrate. Specifically in the presence of a linear chemical gradient that opposes the downhill motion, we found that the droplet always reaches an equilibrium for physically realizable parameter values, which can also exhibit an uphill motion, as suggested by the experiments of Chaudhury \& Whitesides (1992).

Our derivation of a system of IDEs has a direct correspondence with the 2D phase plane for these equations, through which a number of qualitative features of droplet dynamics were extracted, specifically in relation to the topological changes that occur in the phase plane as the inclination angle changed. We demonstrated the existence of a critical inclination angle and confirmed qualitatively the transient dynamics reported in the work of Berejnov \& Thorne (2007). We also examined in detail how the critical angle is affected by topographical and/or chemical heterogeneities and their orientation and deduced an approximate relation for the critical inclination angle, which is valid when we have small-amplitude, single-wavelength, periodic heterogeneities.

We finally note that the present analysis is readily applicable to models that assume the presence of a precursor film as a means to relax the stress singularity of a moving contact line, where the chemical heterogeneities can be incorporated by allowing spatial variations in the Hamaker constant (see, for example, Savva \& Kalliadasis 2011). While our results suggest that many qualitative features are adequately captured with a 2D model, quantitative comparisons with existing experiments cannot be made due to the geometry considered, but we also note the fact that most studies in the literature have focused on large contact angles, i.e. on a regime which is beyond the limit of applicability of the long-wave theory.

\section{Acknowledgements}

We acknowledge financial support from EPSRC Support Fund-Imperial College Internal Funding Opportunities in EPSRC merit, ERC Advanced Grant No. 247031 and EU-FP7 ITN Multiflow.

\section{REFERENCES}

Abramowitz, M. \& Stegun, I. A. 1972 Handbook of Mathematical Functions with Formulas, Graphs, and Mathematical Tables. Dover.

Ben Amar, M., Cummings, L. J. \& Pomeau, Y. 2003 Transition of a moving contact line from smooth to angular. Phys. Fluids 15 (10), 2949-2960. 
Berejnov, V. \& Thorne, R. E. 2007 Effect of transient pinning on stability of drops sitting on an inclined plane. Phys. Rev. E 75, 066308.

Berim, G. O. \& Ruckenstein, E. 2008 Microscopic calculation of the sticking force for nanodrops on an inclined surface. J. Chem. Phys. 129, 114709.

Bertozzi, A. L. \& Brenner, M. P. 1997 Linear stability and transient growth in driven contact lines. Phys. Fluids 9 (3), 530-539.

BiKerman, J. J. 1950 Sliding drops from surfaces of different roughnesses. J. Colloid Sci. 5, 349-359.

Bonn, D., Eggers, J., Indekeu, J., Meunier, J. \& Rolley, E. 2009 Wetting and spreading. Rev. Mod. Phys. 81, 739-805.

Briscoe, B. J. \& GAlvin, K. P. 1991 The sliding of sessile and pendent droplets. The critical condition. Colloids Surf. 52, 219-229.

Brown, R. A., OrR, F. M. Jr \& Scriven, L. E. 1980 Static drop on an inclined plate: an analysis by the finite element method. J. Colloid Interface Sci. 73 (1), 76-87.

Buckingham, R., Shearer, M. \& Bertozzi, A. 2003 Thin films and the Navier slip condition. SIAM J. Appl. Maths 63, 722-744.

Chaudhury, M. K. \& Whitesides, G. M. 1992 How to make water run uphill. Science 256 (5063), 1539-1541.

DAS, A. K. \& DAS, P. K. 2009 Simulation of drop movement over an inclined surface using smoothed particle hydrodynamics. Langmuir 25 (19), 11459-11466.

Diez, J. A., GonzÁlez, A. G. \& KondiC, L. 2012 Instability of a transverse liquid rivulet on an inclined plane. Phys. Fluids 24, 032104.

Diez, J. A. \& Kondic, L. 2001 Contact line instabilities of thin liquid films. Phys. Rev. Lett. 86 (4), 632-635.

Dimitrakopoulos, P. \& Higdon, J. J. L. 1999 On the gravitational displacement of three-dimensional fluid droplets from inclined solid surfaces. J. Fluid Mech. 395, 181-209.

Dupuis, A. \& Yeomans, J. M. 2006 Dynamics of sliding drops on superhydrophobic surfaces. Europhys. Lett. 75 (1), 105-111.

DURBIn, P. A. 1988 Considerations on the moving contact-line singularity, with application to frictional drag on a slender drop. J. Fluid Mech. 197, 157-169.

DUSSAN V, E. B. 1979 On the spreading of liquids on solid surfaces: static and dynamic contact lines. Annu. Rev. Fluid Mech. 11, 371-400.

DUSSAN V, E. B. 1985 On the ability of drops or bubbles to stick to non-horizontal surfaces of solids. Part 2. Small drops or bubbles having contact angles of arbitrary size. J. Fluid Mech. 151, 1-20.

Dussan V, E. B. \& ChOw, R. T.-P. 1983 On the ability of drops or bubbles to stick to non-horizontal surfaces or solids. J. Fluid Mech. 137, 1-29.

ElSherbini, A. I. \& JACOBI, A. M. 2004a Critical contact angles for liquid drops on inclined surfaces. Prog. Colloid Polym. Sci. 128, 57-62.

ElSherbini, A. I. \& JACOBI, A. M. 2004b Liquid drops on vertical and inclined surfaces I. An experimental study on drop geometry. J. Colloid Interface Sci. 273, 556-565.

EXtrand, C. W. \& Kumagai, Y. 1995 Liquid drops on an inclined plane: the relation between contact angles, drop shape and retentive force. J. Colloid Interface Sci. 170, 515-521.

Finn, R. \& Shinbrot, M. 1988 The capillary contact angle, II: the inclined plane. Math. Meth. Appl. Sci. 10, 165-196.

FRENKEL, Y. I. 1948 On the behavior of liquid drops on a solid surface 1. The sliding of drops on an inclined surface. J. Expl Theor. Phys. 18, 659-668.

Furmidge, C. G. L. 1962 The studies at phase interfaces I. The sliding of liquid drops on solid surfaces and a theory for spray retention. J. Colloid Interface Sci. 17, 309-324.

Greenspan, H. P. 1978 On the motion of a small viscous droplet that wets a surface. J. Fluid Mech. 84, 125-143.

Hocking, L. M. 1981 Sliding and spreading of thin two-dimensional drops. Q. J. Mech. Appl. Maths 24, 37-55.

Hocking, L. M. 1983 The spreading of a thin drop by gravity and capillarity. Q. J. Mech. Appl. Maths 36 (1), 55-69. 
HockInG, L. M. 1990 Spreading and instability of a viscous fluid sheet. J. Fluid Mech. 211, 373-392.

Hong, S. D., Ha, M. Y. \& Balachandar, S. 2009 Static and dynamic contact angles of water droplet on a solid surface using molecular dynamics simulation. J. Colloid Interface Sci. 339, $187-195$.

HUPPERT, H. 1982 Flow and instability of a viscous current down a slope. Nature 300, 427-429.

Hyväluoma, J., Koponen, A., Raiskinmäki, P. \& Timonen, J. 2007 Droplets on inclined rough surfaces. Eur. Phys. J. E 23, 289-293.

ILIEV, S. D. 1997 Static drops on an inclined plane: equilibrium modelling and numerical analysis. J. Colloid Interface Sci. 194, 287-300.

KAlliadasis, S. 2000 Nonlinear instability of a contact line driven by gravity. J. Fluid Mech. 413, 355-378.

KIM, H.-Y., LEE, H. J. \& KANG, B. H. 2002 Sliding of liquid drops down an inclined solid surface. J. Colloid Interface Sci. 247, 372-380.

Koh, Y. Y., Lee, Y. C., Gaskell, P. H., Jimack, P. K. \& Thompson, H. M. 2009 Droplet migration: quantitative comparisons with experiment. Eur. Phys. J. Special Topics 166, $117-120$.

KondIC, L. 2003 Instabilities in gravity driven flow of thin liquid films. SIAM Rev. 45 (1), 95-115.

Krasovitski, B. \& MARmur, A. 2005 Drops down the hill: theoretical study of limiting contact angles and the hysteresis range on a tilted plate. Langmuir 21, 3881-3885.

LARKIN, B. K. 1967 Numerical solution of the equation of capillarity. J. Colloid Interface Sci. 23, 305-312.

LAWAL, A. \& Brown, R. A. 1982 The stability of inclined sessile drops. J. Colloid Interface Sci. 89 (2), 346-352.

Le Grand, N., Daerr, A. \& Limat, L. 2005 Shape and motion of drops sliding down an inclined plane. J. Fluid Mech. 541, 293-315.

Limat, L. \& Stone, H. A. 2004 Three-dimensional lubrication model of a contact line corner singularity. Europhys. Lett. 65 (3), 365-371.

LóPeZ, P. G. J., Miksis, M. \& BANKoff, S. G. 1997 Inertial effects on contact line instability in the coating of a dry inclined plate. Phys. Fluids 9 (8), 2117-2183.

Macdougall, G. \& OCKRent, C. 1942 Surface energy relations in liquid/solid systems. I. The adhension of liquids to solids and a new method of determining the surface tension of liquids. Proc. R. Soc. Lond. A 180, 151-173.

Mahadevan, L. \& Pomeau, Y. 1999 Rolling droplets. Phys. Fluids 11 (9), 2449-2453.

Miwa, M., Nakajima, A., Fujishima, A., Hashimoto, K. \& Watanabe, T. 2000 Effects of the surface roughness on sliding angles of water droplets on superhydrophobic surfaces. Langmuir 16, 5754-5760.

Morita, M., Koga, T., Otsuka, H. \& Takahara, A. 2005 Macroscopic wetting anisotropy on the line-patterned surface of fluoroalkylsilane monolayers. Langmuir 21, 911-918.

Pereira, A. \& Kalliadasis, S. 2012 Equilibrium gas-liquid-solid contact angle from density-functional theory. J. Fluid Mech. 692, 53-77.

Pierce, E., Carmona, F. J. \& Amirfazli, A. 2008 Understanding of sliding and contact angle results in tilted plate experiments. Colloids Surf. A 323, 73-82.

Podgorski, T., Flesselles, J.-M. \& Limat, L. 2001 Corners, cusps and pearls in running drops. Phys. Rev. Lett. 87 (3), 036102.

Popova, L. N. 1983 Non-axisymmetric equilibrium shapes of a drop on a plane. Fluid Dyn. 18 (4), 634-637.

QUÉRÉ, D. 1998 Drops at rest on a tilted plane. Langmuir 14, 2213-2216.

RICHARD, D. \& QUÉRÉ, D. 1999 Viscous drops rolling on a tilted non-wettable solid. Europhys. Lett. 48 (3), 286-291.

Rotenberg, Y., Boruvka, L. \& Neumann, A. W. 1984 The shape of non-axisymmetric drops on inclined planar surfaces. J. Colloid Interface Sci. 102 (2), 424-434.

Roura, P. \& ForT, J. 2001 Equilibrium of drops on inclined hydrophilic surfaces. Phys. Rev. E 64, 011601. 
Roura, P. \& FORT, J. 2002 Comment on 'effects of the surface roughness on sliding angles of water droplets on superhydrophobic surfaces'. Langmuir 18, 566-569.

SaVVA, N. \& Kalliadasis, S. 2009 Two-dimensional droplet spreading over topographical substrates. Phys. Fluids 21, 092102.

SaVVA, N. \& Kalliadasis, S. 2011 Dynamics of moving contact lines: A comparison between slip and precursor film models. Europhys. Lett. 94, 64004.

SAVVA, N. \& KALliadASIS, S. 2012 Influence of gravity on the spreading of two-dimensional droplets over topographical substrates. J. Engng Maths 73, 3-16.

Savva, N., Kalliadasis, S. \& Pavliotis, G. A. 2010 Two-dimensional droplet spreading over random topographical substrates. Phys. Rev. Lett. 104, 084501.

Savva, N., Pavliotis, G. A. \& Kalliadasis, S. 2011a Contact lines over random topographical substrates. Part 1. Statics. J. Fluid Mech. 672, 358-383.

Savva, N., Pavliotis, G. A. \& Kalliadasis, S. $2011 b$ Contact lines over random topographical substrates. Part 2. Dynamics. J. Fluid Mech. 672, 384-410.

Servantie, J. \& Müller, M. 2008 Statics and dynamics of a cylindrical droplet under an external body force. J. Chem. Phys. 128, 014709.

Sheng, X. \& ZhANG, J. 2011 Directional motion of water drop on ratchet-like superhydrophobic surfaces. Appl. Surf. Sci. 257, 6811-6816.

SiLvi, N. \& Dussan V, E. B. 1985 On the rewetting of an inclined solid surface by a liquid. Phys. Fluids 28 (1), 5-7.

Snoeijer, J. H., Le Grand-Piteira, N., Limat, L., Stone, H. A. \& Eggers, J. 2007 Cornered drops and rivulets. Phys. Fluids 19, 042104.

Snoeijer, J. H., Rio, E., Le Grand, N. \& Limat, L. 2005 Self-similar flow and contact line geometry at the rear of cornered drops. Phys. Fluids 17, 072101.

Sommers, A. D. \& JACOBI, A. M. 2006 Creating micro-scale surface topology to achieve anisotropic wettability on an aluminum surface. J. Micromech. Microengng 16, 1571-1578.

Sommers, A. D. \& JACOBI, A. M. 2008 Wetting phenomena on micro-grooved aluminum surfaces and modeling of the critical droplet size. J. Colloid Interface Sci. 328, 402-411.

Suzuki, S., Nakajima, A., Tanaka, K., Sakai, M., Hashimoto, A., Yoshida, N., KAMESHIMA, Y. \& OKADA, K. 2008 Sliding behaviour of water droplets on line-patterned hydrophobic surfaces. Appl. Surf. Sci. 254, 1797-1805.

Tadmor, R., Chaurasia, K., Yadav, P. S., Leh, A., Bahadur, P., Dang, L. \& Hoffer, W. R. 2008 Drop retention force as a function of resting time. Langmuir 24, 9370-9374.

Thiele, U., Neuffer, K., Bestehorn, M., Pomeau, Y. \& Velarde, M. G. 2002 Sliding drops on an inclined plane. Colloids Surf. A 206, 87-104.

Thiele, U., Velarde, M. G., Neuffer, K., Bestehorn, M. \& Pomeau, Y. 2001 Sliding drops in the diffuse interface model coupled to hydrodynamics. Phys. Rev. E 64, 061601.

Troian, S. M., Herbolzheimer, E., Safran, S. A. \& Joanny, J. F. 1989 Fingering instabilities of driven spreading films. Europhys. Lett. 19 (1), 25-30.

Vellingiri, R., Savva, N. \& Kalliadasis, S. 2011 Droplet spreading on chemically heterogeneous substrates. Phys. Rev. E 84, 036305.

Yadav, P. S., Bahadur, P., Tadmor, R., Chaurasia, K. \& Leh, A. 2008 Drop retention force as a function of drop size. Langmuir 24, 3181-3184.

Yoshimitsu, Z., Nakajima, A., Watanabe, T. \& Hashimoto, K. 2002 Effects of surface structure on the hydrophobicity and sliding behavior of water droplets. Langmuir 18, $5818-5822$.

Zheng, Y., GAO, X. \& JiAng, L. 2007 Directional adhesion of superhydrophobic butterfly wings. Soft Matt. 3, 178-182. 